\title{
Asymptotics for the biharmonic equation near the tip of a crack
}

\author{
Avner Friedman* $\quad$ Bei Hu ${ }^{\dagger} \quad$ Juan J. L. Velazquez ${ }^{\ddagger}$
}

\section{Introduction}

A mathematical model of a crack in a 2-dimensional uniform elastic medium occupying a bounded domain $\Omega$ consists of the following system (see [9]):

$$
\begin{aligned}
& \varphi \in H^{2}(\Omega), \\
& \Delta^{2} \varphi=0 \quad \text { in } \Omega \backslash \Gamma(t), \\
& \varphi=\frac{\partial \varphi}{\partial n}=0 \quad \text { from both sides of } \Gamma(t), \\
& \varphi=g, \quad \frac{\partial \varphi}{\partial n}=h \quad \text { on } \partial \Omega,
\end{aligned}
$$

where $\varphi=\varphi(x)=\varphi\left(x_{1}, x_{2}\right)$ is the stress function. Here $\Gamma$ is the crack which, for simplicity, we shall take to be a curve of the form

$$
x_{2}=f\left(x_{1}\right), \quad-1 \leqslant x_{1} \leqslant 0
$$

contained in $\Omega$ except for its initial point $(-1, f(-1)$ ), which lies on $\partial \Omega$; we shall also assume, for simplicity, that

$$
f(0)=0, \quad f^{\prime}(0)=0 .
$$

We are interested in the behavior of $\varphi$ near the origin.

From basic work by Kondratév and Oleinik [14] [15] it follows that if $f$ is in $C^{1}\left[-\delta_{0}, 0\right]$ for some $\delta_{0}>0$, then

$$
\varphi \in C^{3 / 2} \text { near the origin, }
$$

and

$$
\begin{aligned}
& |\varphi(x)| \leqslant C|x|^{3 / 2}, \\
& |\nabla \varphi(x)| \leqslant C|x|^{1 / 2} .
\end{aligned}
$$

*IMA, University of Minnesota, Minneapolis, MN 55455

${ }^{\dagger}$ Department of Mathematics, University of Notre Dame, Notre Dame, IN 46556

†Departamento de Matematica Aplicada, Falcultad de Matematicas, Universidad Complutense, 28040 Madrid, Spain 
On the other hand if $f \in C^{\infty}\left[-\delta_{0}, 0\right]$ then, by [5] (and some of the papers referenced therein), $\varphi$ has an asymptotic expansion near the origin. In the special case where

$$
f\left(x_{1}\right) \equiv 0 \quad \text { for }-\delta_{0} \leqslant x_{1} \leqslant 0
$$

the expansion is given explicitly [22] (see also [6; §16] [8; Part II, Chap. 7]) by

$$
\begin{aligned}
\varphi(r, \theta)= & \sum_{k=1}^{\infty} r^{k / 2+1}\left[a_{k} \cos \left(\frac{k}{2}+1\right) \theta+b_{k} \cos \left(\frac{k}{2}-1\right) \theta\right. \\
& \left.+c_{k} \sin \left(\frac{k}{2}-1\right) \theta+d_{k} \sin \left(\frac{k}{2}+1\right) \theta\right],
\end{aligned}
$$

where

$$
\begin{aligned}
& \delta_{0} b_{k+2}+a_{k}=\frac{2}{\pi \delta_{0}^{k / 2+1}} \int_{-\pi}^{\pi} \psi\left(\delta_{0}, \theta\right) \cos \left(\frac{k}{2}+1\right) \theta d \theta \quad \text { for } k \geqslant 2, \\
& \delta_{0}\left(\frac{k}{2}+2\right) b_{k+2}+\left(\frac{k}{2}+1\right) a_{k}=\frac{2}{\pi \delta_{0}^{k / 2}} \int_{-\pi}^{\pi} \psi_{r}\left(\delta_{0}, \theta\right) \cos \left(\frac{k}{2}+1\right) \theta d \theta \quad \text { for } k \geqslant 2
\end{aligned}
$$

(the formulae for $b_{1}, b_{2}, b_{3}, a_{1}$ are little different) and similar relations hold for $c_{k}$, $d_{k}$. Hence we get

$$
\left|a_{k}\right|+\left|b_{k}\right| \leqslant \frac{C}{\delta_{0}^{k}} \int_{-\pi}^{\pi}\left\{\left|\psi\left(\delta_{0}, \theta\right)\right|+\left|\psi_{r}\left(\delta_{0}, \theta\right)\right|\right\} d \theta,
$$

and the same inequality holds for $\left|c_{k}\right|+\left|d_{k}\right|$. It follows that the series (1.10) is uniformly convergent for $0 \leqslant r \leqslant \theta \delta_{0}$, for any $\theta<1$.

From (1.10) we get

$$
\begin{array}{r}
\varphi(r, \theta)=\quad A_{1} r^{3 / 2} B_{1}(\theta)+A_{2} r^{3 / 2} B_{2}(\theta)+A_{3} r^{2} B_{3}(\theta) \\
+A_{4} r^{5 / 2} B_{4}(\theta)+A_{5} r^{5 / 2} B_{5}(\theta)+O\left(r^{3}\right),
\end{array}
$$

where

$$
\begin{gathered}
B_{1}(\theta)=\cos \frac{3}{2} \theta+3 \cos \frac{1}{2} \theta, \quad B_{2}(\theta)=\sin \frac{3}{2} \theta+\sin \frac{1}{2} \theta, \quad B_{3}(\theta)=\sin ^{2} \theta, \\
B_{4}(\theta)=\cos \frac{5}{2} \theta-5 \cos \frac{1}{2} \theta, \quad B_{5}(\theta)=\sin \frac{5}{2} \theta-\sin \frac{1}{2} \theta ;
\end{gathered}
$$

note that $r^{2} B_{3}(\theta)=x_{2}^{2}$.

The main purpose of this paper is to establish asymptotic expansions of up to order $r^{3-\eta}$, under very weak assumptions on the regularity of $f\left(x_{1}\right)$. Our results are:

(i) If $f \in C^{1+\alpha}\left[-\delta_{0}, 0\right]$, then

$$
\varphi(x)=A_{1} r^{3 / 2} B_{1}(\theta)+A_{2} r^{3 / 2} B_{2}(\theta)+O\left(r^{2-\eta}\right)
$$

for any $\eta>0$ such that $\alpha+\eta>1 / 2$. 
(ii) If $f \in C^{2+\alpha}\left[-\delta_{0}, 0\right]$, then

$$
\begin{gathered}
\varphi(r, \theta)=A_{1} r^{3 / 2} B_{1}(\theta)+A_{2} r^{3 / 2} B_{2}(\theta)+A_{3} r^{2} B_{3}(\theta)+A_{4} r^{5 / 2} B_{4}(\theta) \\
+A_{5} r^{5 / 2} B_{5}(\theta)-2 A_{2} r^{5 / 2} f^{\prime \prime}(0) \cos \frac{1}{2} \theta+O\left(r^{3-\eta}\right)
\end{gathered}
$$

for any $\eta>0$ such that $\alpha+\eta>1 / 2$.

Note that if $f^{\prime \prime}(0)=0$, then the expansion (1.14) agrees with that of (1.11).

The first two terms in the above expansion are called, in fractural mechanics, the stress intensity factors; or mode I and mode II of fracture [16; p. 24].

The proof of the estimates (i), (ii) require maximum principles for biharmonic solutions in a domain whose boundary has a singular point. Such estimates are established in $\S 2$; for related results see Remark 2.3. In $\S 3$ we give a proof of (i) and, in $\S 5$, a proof of (ii). In $\S 4$ we derive an additional regularity result for $\varphi$, near the tip $O=(0,0)$, for $f$ in $C^{1+\alpha}$, namely:

$$
\frac{\varphi(x)}{|x|^{3 / 2}} \leqslant C\left(|x|^{2 \alpha}+\frac{d^{2}(x)}{|x|^{2}}\right) \text { near the origin, }
$$

where $d(x)=\operatorname{dist}(x, \Gamma)$. This improves the inequality that can be obtained by sub-Schauder $(2-\eta)$-estimates (stated in $\S 9$, Example 2), if $|x|^{\alpha+\eta^{\prime}}<d(x) /|x| \ll 1$ for some $\eta^{\prime}>0$.

The remaining part of the paper is concerned with an application of some of the above results to the crack propagation problem, where the tip of the crack is moving in time according to (see [9])

$$
\dot{X}(t)=h(|J(X(t))|)|J(X(t))|
$$

where $X(t)$ is the tip of the crack at time $t, h(s)$ is a given function, and $J(X(t))$ is the limit of $J$-integrals taken along circles that shrink to $X(t)$. The model is described in $\S 6$. In $\S 7$ we prove that the crack propagation problem with $C^{1+\alpha}$ crack is equivalent to the following geometric problem:

Find an extension $x_{2}=f\left(x_{1}\right),-1 \leqslant x_{1} \leqslant \tau(\tau>0)$ of $\Gamma$ which is $C^{1+\alpha}$ such that, at each intermediate value $x_{1}=s$, the coefficient $A_{2}=A_{2}(s)$ in the asymptotic expansion about the tip $X(s)=(s, f(s))$ satisfies:

$$
A_{2}(s) \equiv 0 \text {. }
$$

In $\S 8$ we make a few comments on this problem, which we hope to pursue in a future work. The paper concludes with an appendix in which we have assembled several sub-Schauder estimates used in this paper.

\section{Maximum principles}

In this section we establish estimates for solutions of $\Delta^{2} \varphi=f$ in a bounded domain $\Omega$ in terms of the supremum of the boundary values of $\varphi$ and $\partial \varphi / \partial \nu$ on $\partial \Omega$. When $\partial \Omega$ is $C^{4}$ such an estimate is well known:

$$
\|\varphi\|_{W^{1, \infty}(\Omega)} \leqslant C\left(\|\varphi\|_{L^{\infty}(\partial \Omega)}+\|\nabla \varphi\|_{L^{\infty}(\partial \Omega)}+\|f\|_{L^{1}(\Omega)}\right) .
$$


This estimate was derived by Miranda [18] when $\Omega$ is 2-dimensional, and by Agmon [2] for $\Omega$ which is $n$-dimensional; Agmon has actually extended the results to elliptic operators of any order with variable coefficients.

For our purposes we need to deal with domains whose boundary has a singularity as, for example, in Figure 1 below. However we begin with a local version of the type (2.1).

Let $\Omega$ be a bounded $n$-dimensional domain, $S$ an open subset of $\partial \Omega, S$ in $C^{4}$, and $D$ a subdomain of $\Omega$ such that

$$
\bar{D} \subset \Omega \cup S_{0} \quad \text { where } S_{0} \subset \partial \Omega, \bar{S}_{0} \subset \operatorname{int} S
$$

Lemma 2.1 Under the above assumptions there is a constant $C$ such that for any function $\varphi$ in $L^{p}(\Omega) \cap C^{1}(\Omega \cup S), p>1$, if

$$
\Delta^{2} \varphi=f \quad \text { in } \Omega
$$

then

$$
\|\varphi\|_{W^{1, \infty}(D)} \leqslant C\left(\|\varphi\|_{L^{\infty}(S)}+\|\nabla \varphi\|_{L^{\infty}(S)}+\|\varphi\|_{L^{p}(\Omega)}+\|f\|_{L^{1}(\Omega)}\right)
$$

Proof. Set

$$
\Lambda=\|\varphi\|_{L^{\infty}(S)}+\|\nabla \varphi\|_{L^{\infty}(S)}+\|\varphi\|_{L^{p}(\Omega)}+\|f\|_{L^{1}(\Omega)} .
$$

Without loss of generality we may assume that $f=0$; otherwise we subtract from $\varphi$ the special solution of $(2.2)$

$$
\frac{1}{8 \pi} \int_{\Omega}|x-y|^{2} \log \frac{1}{|x-y|} f(y) d y .
$$

Introduce domains $D_{1} \subset D_{2} \subset \Omega$ with

$$
\begin{aligned}
& \bar{D} \subset D_{1}, \quad \bar{D}_{1} \subset D_{2}, \quad \bar{D}_{2} \subset \Omega \cup S_{0} \\
& \partial D \cap S \subset \operatorname{int}\left(\partial D_{1} \cap S\right), \quad \partial D_{1} \cap S \subset \operatorname{int}\left(\partial D_{2} \cap S\right) .
\end{aligned}
$$

Take a $C^{\infty}$ function $\zeta$ such that

$$
\begin{array}{ll}
\zeta=1 & \text { in } \bar{D}_{1}, \\
\zeta=0 & \text { in } \bar{\Omega} \backslash D_{2}
\end{array}
$$

and introduce a domain $\widetilde{D}$ with $C^{4}$ boundary such that

$$
D_{1} \subset \tilde{D} \subset D_{2}, \quad \text { and } \zeta \equiv 0 \quad \text { in } \Omega \backslash \widetilde{D}
$$

Consider the function $v=\zeta \varphi$. It satisfies

$$
\Delta^{2} v=\sum_{|\alpha| \leqslant 3} \zeta_{\alpha} D^{\alpha} \varphi
$$


and

$$
v=\frac{\partial v}{\partial \nu}=0 \quad \text { on } \partial \widetilde{D} \backslash S
$$

Write $v=v_{1}+v_{2}$ where

$$
\begin{aligned}
& \Delta^{2} v_{1}=0 \quad \text { in } \tilde{D}, \\
& v_{1}=v, \quad \frac{\partial v_{1}}{\partial \nu}=\frac{\partial v}{\partial \nu} \quad \text { on } \partial \widetilde{D} .
\end{aligned}
$$

By the Miranda-Agmon maximum principle

$$
\left\|v_{1}\right\|_{W^{1, \infty}(\widetilde{D})} \leqslant C\left(\|\zeta \varphi\|_{L^{\infty}(\partial \widetilde{D})}+\|\nabla(\zeta \varphi)\|_{L^{\infty}(\partial \widetilde{D})}\right) \leqslant C\left(\|\varphi\|_{L^{\infty}(S)}+\|\nabla \varphi\|_{L^{\infty}(S)}\right) .
$$

To estimate $v_{2}$ we apply Theorem 8.1 of [1] which says: If for any $w \in C^{4}(\widetilde{D})$ with $w=\frac{\partial w}{\partial \nu}=0$ on $\partial \widetilde{D}$ there holds

$$
\left|\int_{\widetilde{D}} v_{2} \Delta^{2} w d x\right| \leqslant \lambda|w|_{W^{4-k, p^{\prime}}(\widetilde{D})}\left(\frac{1}{p}+\frac{1}{p^{\prime}}=1\right)
$$

for some integer $k, 0 \leqslant k \leqslant 4$, then

$$
\left|v_{2}\right|_{W^{k, p}(\tilde{D})} \leqslant C \lambda
$$

here $v_{2}$ is an arbitrary function (say in $L^{p}(\widetilde{D})$ ), $\lambda$ is a constant depending on $v_{2}$, and $C$ is a constant independent of $v_{2}, \lambda$.

We shall apply this result to the function $v_{2}$ defined above as $v-v_{1}$. By integration by parts,

$$
\begin{aligned}
\int_{\widetilde{D}} v_{2} \Delta^{2} w d x & =\int_{\widetilde{D}} \Delta^{2} v_{2} \cdot w d x=\int_{\widetilde{D}} \sum_{|\alpha| \leqslant 3} \zeta_{\alpha} D^{\alpha} \varphi \cdot w d x \\
& =\int_{\widetilde{D}} \sum_{|\alpha| \leqslant 3} \varphi(-1)^{\alpha} D^{\alpha}\left(\zeta_{\alpha} w\right) d x
\end{aligned}
$$

so that (2.6) holds with $k=1, \lambda=C\|\varphi\|_{L^{p}}$. Consequently, by (2.7),

$$
\left\|v_{2}\right\|_{W^{1, p}(\widetilde{D})} \leqslant C\|\varphi\|_{L^{p}(\Omega)}
$$

Combining this with (2.5) we get

$$
\|\varphi\|_{W^{1, p}(\widetilde{D})} \leqslant\|v\|_{W^{1, p}(\widetilde{D})} \leqslant C \Lambda
$$

We can now repeat the above argument with the smaller domains $D_{1}, \widetilde{D}, D_{2}$ (still containing $D)$ and $k=2$ in (2.6). We get

$$
\left\|v_{1}\right\|_{W^{1, \infty}(\widetilde{D})}+\left\|v_{2}\right\|_{W^{2, p}(\widetilde{D})} \leqslant C \Lambda
$$


If $p>n$, then by Sobolev embedding we deduce that

$$
\left\|v_{2}\right\|_{W^{1, \infty}(\widetilde{D})} \leqslant C \Lambda
$$

and (2.3) follows. If however $p \leqslant n$, we repeat the above process with a larger value of $p$; in fact, if $p<n$, then

$$
\varphi \in L^{q}(\widetilde{D}), \quad \text { and }\|\varphi\|_{L^{q}(\widetilde{D})} \leqslant C \Lambda, \quad \text { where } \frac{1}{q}=\frac{1}{p}-\frac{1}{n}
$$

whereas if $p=n$, then

$$
\varphi \in L^{q}(\widetilde{D}), \quad \text { and }\|\varphi\|_{L^{q}(\widetilde{D})} \leqslant C \Lambda, \forall q<+\infty .
$$

After a finite number of steps the proof of (2.3) is completed.

We shall now specialize to 2-dimensional domains whose boundary has a singular point. The method of Miranda-Agmon does not extend to such domains, and, in fact, our estimates will also be quite different.

Let $\Omega_{\omega}$ be a domain shown in Figure 1, consisting of two line segments $|\theta|=\omega / 2(\pi<$ $\omega \leqslant 2 \pi)$, connected by an arc on the circle $|x|=1$, and regularized around $|\theta|=\omega / 2,|x|=1$ so that $\partial \Omega_{\omega} \backslash\{0\} \in C^{\infty}$.

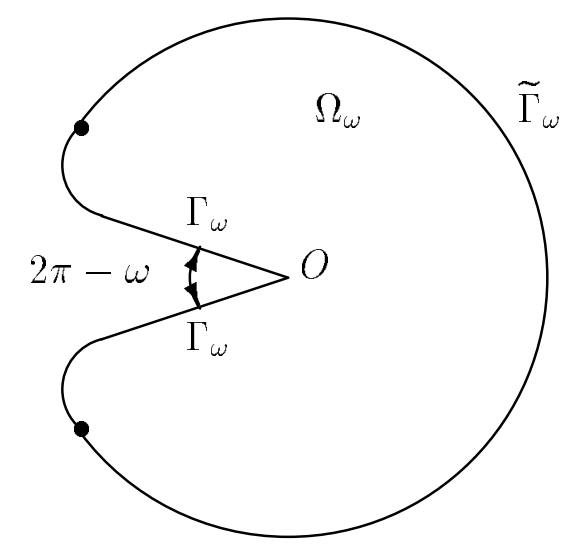

Fig. 1

Then $\partial \Omega_{\omega}=\Gamma_{\omega} \cup \widetilde{\Gamma}_{\omega}$, where $\widetilde{\Gamma}_{\omega}=\partial \Omega_{\omega} \backslash \Gamma_{\omega}=\partial \Omega_{\omega} \cap\{|x|=1\}$ and $\Gamma_{\omega}$ consists of the two line segments $\theta= \pm \omega / 2$ initiating at the origin with two small smooth arcs attached at each endpoint.

Theorem 2.2 Suppose that $\varphi \in H^{2}\left(\Omega_{\omega}\right)$,

$$
\begin{aligned}
& \Delta^{2} \varphi=f \quad \text { in } \Omega_{\omega} \\
& \varphi=\frac{\partial \varphi}{\partial n}=0 \quad \text { on } \partial \Omega_{\omega} \backslash \Gamma_{\omega} .
\end{aligned}
$$


Then, for any $p>2, \sigma>0, \mu<2 / p$, there exists a constant $C=C_{p, \sigma, \mu}>0$, depending only on the regularity of $\partial \Omega_{\omega} \cap\{|x| \geqslant 1 / 2\}$ (but not on the angle size $\omega$ ), such that

$$
\begin{aligned}
\left(\int_{\Omega_{\omega}}|x|^{-p \mu}|\varphi|^{p} d x\right)^{1 / p} & \leqslant C\left[\left\{\int_{\Gamma_{\omega}}\left(|x|^{-(1 / 2+2 / p+\sigma)}|\varphi(x)|\right)^{p / 2} d S\right\}^{2 / p}\right. \\
+ & \left.\int_{\Gamma_{\omega}}|x|^{-1 / 2}\left|\frac{\partial \varphi(x)}{\partial n}\right| d S+\int_{\Omega_{\omega}}|x|^{3 / 2}|f(x)| d x\right] .
\end{aligned}
$$

Proof. Define $u$ to be the solution of the following problem:

$$
\begin{aligned}
& u \in H^{2}\left(\Omega_{\omega}\right), \\
& \Delta^{2} u=g \text { in } \Omega_{\omega}, \\
& u=\frac{\partial u}{\partial n}=0 \text { on } \partial \Omega_{\omega},
\end{aligned}
$$

where $g$ is any function in $L^{2}\left(\Omega_{\omega}\right)$. By integration by parts (cf. [14; equation (5)])

$$
\int_{\Omega_{\omega}} E[u] d x=\int_{\Omega_{\omega}} u g d x \leqslant C\|u\|_{L^{\infty}\left(\Omega_{\omega}\right)}\|g\|_{L^{1}\left(\Omega_{\omega}\right)}
$$

where $E[u]=\sum_{i, j=1}^{2}\left(\frac{\partial^{2} u}{\partial x_{i} \partial x_{j}}\right)^{2}, 1 / q+1 / \widetilde{q}=1,1<q<2$. By embedding,

$$
\|u\|_{L^{\infty}\left(\Omega_{\omega}\right)} \leqslant C\left(\int_{\Omega_{\omega}} E[u] d x\right)^{1 / 2}
$$

and hence

$$
\int_{\Omega_{\omega}} E[u] d x \leqslant C\|g\|_{L^{1}\left(\Omega_{\omega}\right)}^{2} .
$$

We shall now use the inequalities (40) and (47) of [14] (see also $§ 9$ ):

$$
\begin{aligned}
& |u(x)|^{2} \leqslant C|x|^{2+2 \delta}\left(\|g\|_{L^{1}\left(\Omega_{\omega}\right)}^{2}+\int_{\Omega_{\omega}} E[u] d x\right), \\
& |\nabla u(x)|^{2} \leqslant C|x|^{2 \delta}\left(\|g\|_{L^{1}\left(\Omega_{\omega}\right)}^{2}+\int_{\Omega_{\omega}} E[u] d x\right)
\end{aligned}
$$

where $\delta=\delta(\widetilde{\omega})$ is the solution of

$$
\sin ^{2}(\widetilde{\omega} \delta)=\delta^{2} \sin ^{2} \tilde{\omega}, \quad 0<\tilde{\omega} \delta(\widetilde{\omega}) \leqslant \pi, \quad \delta(\widetilde{\omega}) \geqslant \frac{1}{2}
$$

and $\widetilde{\omega}$ is any constant such that $\omega \leqslant \widetilde{\omega}$ and $\pi<\tilde{\omega} \leqslant 2 \pi$; it is easy to verify that there exists a unique such $\delta=\delta(\widetilde{\omega})$. We take $\widetilde{\omega}=2 \pi$ so that $\delta(\widetilde{\omega})=1 / 2$. If we substitute (2.13) into these inequalities, we obtain

$$
\begin{aligned}
& |u(x)|^{2} \leqslant C|x|^{3}\|g\|_{L^{1}\left(\Omega_{\omega}\right)}^{2}, \\
& |\nabla u(x)|^{2} \leqslant C|x|\|g\|_{L^{1}\left(\Omega_{\omega}\right)}^{2} .
\end{aligned}
$$


To obtain the estimates for the second and third order derivatives for $u$, we introduce the scaling

$$
u_{\varepsilon}(x)=u(\varepsilon x) \quad \text { for } 1 \leqslant|x| \leqslant 4 \text {. }
$$

Then

$$
\begin{aligned}
& \Delta^{2} u_{\varepsilon}=\varepsilon^{4} g(\varepsilon x) \quad \text { in } D=\left\{1<|x|<4,-\frac{\omega}{2}<\theta<\frac{\omega}{2}\right\} \\
& u_{\varepsilon}=\frac{\partial u_{\varepsilon}}{\partial n}=0 \quad \text { on } \partial D .
\end{aligned}
$$

By interior-boundary $L^{\widetilde{p}}$ estimates,

$$
\begin{aligned}
\left\|u_{\varepsilon}\right\|_{W^{4, \tilde{p}}\left(D_{0}\right)} & \leqslant C_{\widetilde{p}}\left[\max _{D}\left|u_{\varepsilon}(x)\right|+\varepsilon^{4}\left(\int_{D}|g|^{\widetilde{p}}(\varepsilon x) d x\right)^{1 / \widetilde{p}}\right] \\
& \leqslant C_{\widetilde{p}}\left(\varepsilon^{3 / 2}\|g\|_{L^{1}\left(\Omega_{\omega}\right)}+\varepsilon^{4-2 / \widetilde{p}}\|g\|_{L^{\tilde{p}}\left(\Omega_{\omega} \cap\{\varepsilon<|x|<4 \varepsilon\}\right)}\right),
\end{aligned}
$$

where $D_{0}=D \cap\{2<|x|<3\}, 1 / p+1 / \tilde{p}=1$. By embedding

$$
\begin{aligned}
& \left\|u_{\varepsilon}\right\|_{W^{3, r}\left(D_{0} \cap \Gamma\right)} \leqslant C_{\widetilde{p} \varepsilon^{3 / 2}}\left(\|g\|_{L^{1}\left(\Omega_{\omega}\right)}+\left\||x|^{5 / 2-2 / \widetilde{p}} g\right\|_{L^{\tilde{p}}\left(\Omega_{\omega} \cap\{\varepsilon<|x|<4 \varepsilon\}\right)}\right), \\
& \left\|u_{\varepsilon}\right\|_{W^{2, \infty}\left(D_{0} \cap \Gamma\right)} \leqslant C_{\tilde{p} \varepsilon^{3 / 2}}\left(\|g\|_{L^{1}\left(\Omega_{\omega}\right)}+\left\||x|^{5 / 2-2 / \widetilde{p}} g\right\|_{L^{\tilde{p}}\left(\Omega_{\omega} \cap\{\varepsilon<|x|<4 \varepsilon\}\right)}\right),
\end{aligned}
$$

where $\Gamma=\{\theta= \pm \omega / 2\}, r=\tilde{p} /(2-\tilde{p})=p /(p-2)$, and so $1 / r+1 / r^{\prime}=1$ for $r^{\prime}=p / 2$. Rewriting this in terms of the original variables, we have

$$
\begin{aligned}
& \left(\int_{\{2 \varepsilon<|x|<3 \varepsilon\} \cap \Gamma_{\omega}}\left|D^{3} u(x)\right|^{r} d S\right)^{1 / r} \leqslant C \varepsilon^{-3 / 2+1 / r}\left(\|g\|_{L^{1}\left(\Omega_{\omega}\right)}+\left\||x|^{5 / 2-2 / \tilde{p}} g\right\|_{L^{\tilde{p}}\left(\Omega_{\omega}\right)}\right), \\
& \max _{\{2 \varepsilon<|x|<3 \varepsilon\} \cap \Gamma_{\omega}}\left|D^{2} u(x)\right| \leqslant C \varepsilon^{-1 / 2}\left(\|g\|_{L^{1}\left(\Omega_{\omega}\right)}+\left\||x|^{5 / 2-2 / \tilde{p}} g\right\|_{L^{\tilde{p}}\left(\Omega_{\omega}\right)}\right) .
\end{aligned}
$$

Setting $K=\left(\|g\|_{L^{1}\left(\Omega_{\omega}\right)}+\left\||x|^{5 / 2-2 / \widetilde{p}} g\right\|_{L^{\tilde{p}}\left(\Omega_{\omega}\right)}\right)$, it then follows that

$$
\begin{aligned}
& \int_{\left.\{2 \varepsilon<|x|<3 \varepsilon\} \cap \Gamma_{\omega}\right\}}\left(|x|^{3 / 2-1 / r+\sigma}\left|D^{3} u(x)\right|\right)^{r} d S \leqslant\left(C \varepsilon^{\sigma} K\right)^{r}, \\
& \left|D^{2} u(x)\right| \leqslant C|x|^{-1 / 2} K .
\end{aligned}
$$

Letting $\varepsilon_{j}=(3 / 2)^{-j}$ and summing over $j$, we conclude that

$$
\left(\int_{\{|x|<1 / 2\} \cap \Gamma_{\omega}}\left(|x|^{3 / 2-1 / r+\sigma}\left|D^{3} u(x)\right|\right)^{r} d S\right)^{1 / r} \leqslant C K .
$$

Inequality (2.18) is clearly valid if we integrate also over $\Gamma_{\omega} \backslash\{|x|<1 / 2\}$, and similarly (2.17) is valid over all of $\Gamma_{\omega}$.

We now multiply equation (2.11) by $\varphi$ and integrate by parts to obtain

$$
\int_{\Omega_{\omega}} \varphi \cdot g d x=\int_{\Gamma_{\omega}}\left(\varphi \frac{\partial \Delta u}{\partial n}-\frac{\partial \varphi}{\partial n} \Delta u\right) d S+\int_{\Omega_{\omega}} u \cdot f d x
$$




$$
\begin{aligned}
\leqslant\left\{\int_{\Gamma_{\omega}}\left(|x|^{3 / 2-1 / r+\sigma}\left|D^{3} u(x)\right|\right)^{r} d S\right\}^{1 / r}\left\{\int_{\Gamma_{\omega}}\left(|x|^{-(3 / 2-1 / r+\sigma)}|\varphi(x)|\right)^{r^{\prime}} d x\right\}^{1 / r^{\prime}} \\
+\sup \left(|x|^{1 / 2}\left|D^{2} u(x)\right|\right) \int_{\Gamma_{\omega}}|x|^{-1 / 2}\left|\frac{\partial \varphi(x)}{\partial n}\right| d x \\
+\sup \left(|x|^{-3 / 2}|u(x)|\right) \int_{\Omega_{\omega}}|x|^{3 / 2}|f(x)| d x \\
\leqslant C K\left[\left\{\int_{\Gamma_{\omega}}\left(|x|^{-(1 / 2+2 / p+\sigma)}|\varphi(x)|\right)^{p / 2} d S\right\}^{2 / p}\right. \\
\left.+\int_{\Gamma_{\omega}}|x|^{-1 / 2}\left|\frac{\partial \varphi(x)}{\partial n}\right| d S+\int_{\Omega_{\omega}}|x|^{3 / 2}|f(x)| d x\right]
\end{aligned}
$$

Since, by Hölder's inequality,

$$
K \leqslant C_{\mu}\left\||x|^{\mu} g\right\|_{L^{q}\left(\Omega_{\omega}\right)}
$$

for any $\mu<2 / p$, the assertion (2.10) follows immediately by duality.

Remark 2.1. Let $\lambda=$ right-hand side of (2.10). Then, by Theorem 2.2,

$$
\int_{\Omega_{\omega}}|x|^{-\mu p}|\varphi(x)|^{p} d x \leqslant C \lambda^{p} .
$$

Take $\varphi_{\varepsilon}(x)=\varphi(\varepsilon x)$ as before. Then

$$
\int_{\{1<|x|<4\}}\left|\varphi_{\varepsilon}(x)\right|^{p} d x \leqslant \frac{1}{\varepsilon^{2}} \int_{\Omega_{\omega} \cap\{\varepsilon<|y|<4 \varepsilon\}}|\varphi(y)|^{p} d y \leqslant C \lambda^{p} \varepsilon^{\mu p-2} .
$$

and, by Lemma 2.1, for $|x|=2$,

$$
\begin{aligned}
\left|\varphi_{\varepsilon}(x)\right|+\left|\nabla \varphi_{\varepsilon}(x)\right| & \leqslant C\left\{\lambda \varepsilon^{\mu-2 / p}+\max _{\Gamma_{\omega} \cap\{1<|x|<4\}}\left[\left|\varphi_{\varepsilon}(x)\right|+\left|\nabla \varphi_{\varepsilon}(x)\right|\right]\right\} \\
& \leqslant C\left\{\lambda \varepsilon^{\mu-2 / p}+\max _{\Gamma_{\omega} \cap\{\varepsilon<|y|<4 \varepsilon\}}[|\varphi(y)|+|y||\nabla \varphi(y)|]\right\} .
\end{aligned}
$$

Rewriting this in terms of the original variables, we get

$$
\begin{aligned}
& |\varphi(x)| \leqslant C \lambda|x|^{\mu-2 / p}+C \max _{\Gamma_{\omega} \cap\{|x| / 2<|y|<2|x|\}}[|\varphi(y)|+|y||\nabla \varphi(y)|], \\
& |\nabla \varphi(x)| \leqslant C \lambda|x|^{\mu-1-2 / p}+C_{\Gamma_{\omega} \cap\{|x| / 2<|y|<2|x|\}}\left[|y|^{-1}|\varphi(y)|+|\nabla \varphi(y)|\right] .
\end{aligned}
$$

Later on we shall also need a maximum principle in a domain $\Omega^{\delta}$, shown in Figure 2; it is bounded by a circle $|x|=1$, a circle $\Gamma_{\delta}:|x-(\delta, 0)|=\delta$, and a curve: $x_{2}=f\left(x_{1}\right),-1<x_{1}<\delta$. We assume that $f\left(x_{1}\right)$ is in $C^{1+\alpha}$ for $-1<x_{1}<\delta$, and that $f(0)=0, f^{\prime}(0)=0$. 


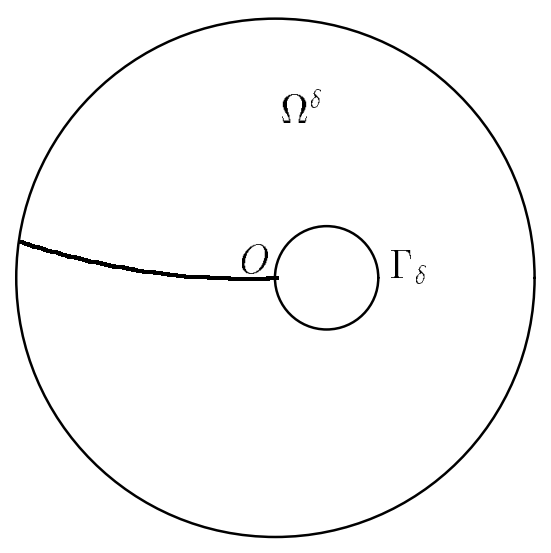

Fig. 2

Theorem 2.3 Let the foregoing assumptions on $\Omega^{\delta}, \Gamma_{\delta}$ hold, and suppose that $\varphi \in H^{2}\left(\Omega^{\delta}\right)$,

$$
\begin{aligned}
& \Delta^{2} \varphi=f \quad \text { in } \Omega^{\delta}, \\
& \varphi=\varphi_{n}=0 \quad \text { on } \partial \Omega^{\delta} \backslash \Gamma_{\delta} .
\end{aligned}
$$

Then, for any $p>2, \sigma>0, \mu<2 / p$, there exists a constant $C=C_{p, \sigma, \mu}>0$ independent of $\delta$ such that

$$
\begin{aligned}
\left(\int_{\Omega^{\delta}}|x|^{-p \mu}|\varphi|^{p} d x\right)^{1 / p} & \leqslant C\left[\left\{\int_{\Gamma_{\delta}}\left(|x|^{-(1 / 2+2 / p+\sigma)}|\varphi(x)|\right)^{p / 2} d S\right\}^{2 / p}\right. \\
& \left.+\int_{\Gamma_{\delta}}|x|^{-1 / 2}\left|\frac{\partial \varphi(x)}{\partial n}\right| d S+\int_{\Omega^{\delta}}|x|^{3 / 2}|f(x)| d x\right]
\end{aligned}
$$

Proof. The proof is similar to that for Theorem 2.2. Denote by $u$ the solution of the following problem

$$
\begin{aligned}
& u \in H^{2}\left(\Omega^{\delta}\right), \\
& \Delta^{2} u=g \quad \text { in } \Omega^{\delta}, \quad(p>2) \\
& u=\frac{\partial u}{\partial n}=0 \quad \text { on } \partial \Omega^{\delta} .
\end{aligned}
$$

As before, we obtain

$$
\|u\|_{L^{\infty}\left(\Omega^{\delta}\right)}+\left(\int_{\Omega^{\delta}} E[u] d x\right)^{1 / 2} \leqslant C\|g\|_{L^{1}\left(\Omega^{\delta}\right)} .
$$

From (40) of [11],

$$
|u(x)| \leqslant C|x|^{3 / 2}\|g\|_{L^{1}\left(\Omega^{\delta}\right)} \quad \text { in } \Omega^{\delta},
$$

where the constant $C$ is independent of $\delta$. 
Now we proceed as in the derivation of $(2.16),(2.17)$ to derive, for $\varepsilon<\delta$, the inequalities

$$
\begin{aligned}
& \int_{\left.\{2 \varepsilon<|x|<3 \varepsilon\} \cap \Gamma_{\delta}\right\}}\left(|x|^{3 / 2-1 / r+\sigma}\left|D^{3} u(x)\right|\right)^{r} d S \leqslant\left(C \varepsilon^{\sigma} K\right)^{r}, \\
& \left|D^{2} u(x)\right| \leqslant C \varepsilon^{-1 / 2} K \quad \text { for } x \in\{2 \varepsilon<|x|<3 \varepsilon\} \cap \Gamma_{\delta},
\end{aligned}
$$

where $K$ is defined as before as above but with $\Omega_{\omega}$ replaced by $\Omega^{\delta}$. (The assumption $\varepsilon<\delta$ is necessary to ensure that the rescaled function satisfies the equation in a domain with uniformly smooth boundary.) The rest of the proof is the same as in Theorem 2.2.

Finally, we prove a maximum principle for the domain $\Omega^{\delta, m}$, bounded by a circle $|x|=1$, and $\Gamma^{\delta, m}$, where $m \geqslant 2 \delta$, and

$$
\Gamma^{\delta, m}=\left\{\left(x_{1}, x_{2}\right) \mid \frac{1}{\delta}\left(x_{1}, x_{2}\right) \in \Gamma^{1, m / \delta}\right\}
$$

and $\Gamma^{1, m / \delta}$ is a curve consisting of two semi-circles centered at $(1,0)$ and $(\mathrm{m} / \delta, 0)$ of radius 1 , connected by two line segments, regularized near four points $(1,1),(1,-1),(m / \delta, 1)$, $(m / \delta,-1)$ so that $\Gamma^{1, m / \delta} \in C^{5}$.

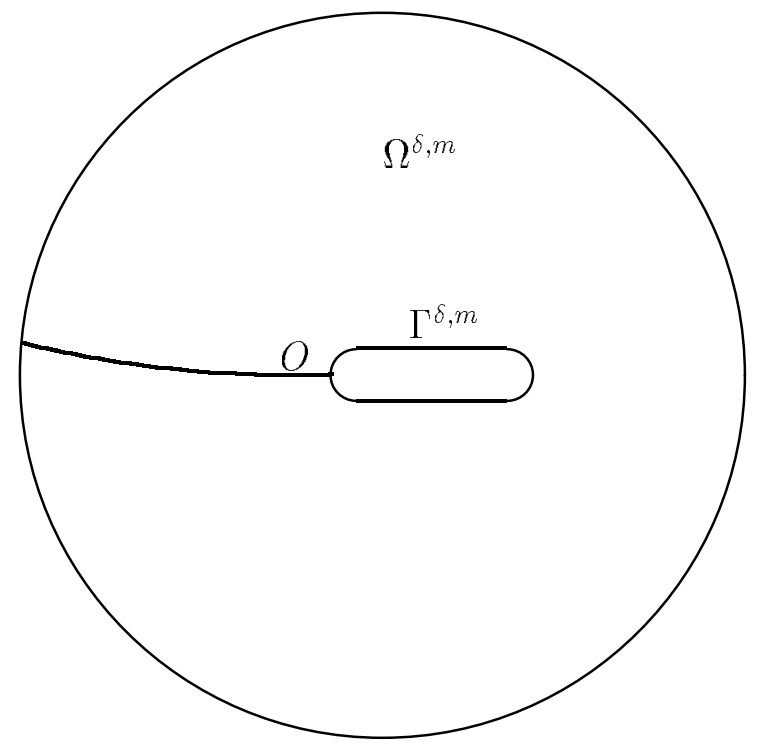

Fig. 3

We follow the proof of Theorem 2.2. First, it is clear that the estimates $(2.16),(2.17)$ are still valid in the region $\{2 \varepsilon<|x|<3 \varepsilon\}$ for $\varepsilon<\delta$. Next, for $|x| \geqslant 3 \delta$, the argument of Theorem 2.2 leads to

$$
\begin{aligned}
& \int_{\{2 \varepsilon<d(x)<3 \varepsilon\} \cap \Gamma^{\delta, m}}\left(d(x)^{3 / 2-1 / r+\sigma}\left|D^{3} u(x)\right|\right)^{r} d S \leqslant\left(C \varepsilon^{\sigma} \widetilde{K}\right)^{r}, \\
& \left|D^{2} u(x)\right| \leqslant C d(x)^{-1 / 2} \widetilde{K} \quad \text { for }|x| \geqslant 3 \delta .
\end{aligned}
$$


where

$$
d(x)=\min (|x|,|x-(m, 0)|), \quad \delta \leqslant \varepsilon<m,
$$

and $\widetilde{K}$ is defined as $K$ but with $|x|^{5 / 2-2 / q}$ replaced by $d(x)^{5 / 2-2 / q}$ and with $\Omega_{\omega}$ replaced by $\Omega^{\delta, m}$.

Now we can take $\varepsilon=\varepsilon_{j}$ as before to conclude:

Theorem 2.4 Suppose that $\varphi \in H^{2}\left(\Omega^{\delta, m}\right)$,

$$
\begin{aligned}
& \Delta^{2} \varphi=f \quad \text { in } \Omega^{\delta, m}, \\
& \varphi=\varphi_{n}=0 \quad \text { on } \partial \Omega^{\delta, m} \backslash \Gamma^{\delta, m} .
\end{aligned}
$$

Then, for any $p>2, \sigma>0 \mu>0$, there exists a constant $C=C_{p, \sigma, \mu}>0$ independent of $\delta$ and $m$ such that

$$
\begin{aligned}
\left(\int_{\Omega^{\delta, m}} d(x)^{-p \mu}|\varphi|^{p} d x\right)^{1 / p} & \leqslant C\left[\left\{\int_{\Gamma^{\delta, m}}\left(d(x)^{-(1 / 2+2 / p+\sigma)}|\varphi(x)|\right)^{p / 2} d S\right\}^{2 / p}\right. \\
& \left.+\int_{\Gamma^{\delta, m}} d(x)^{-1 / 2}\left|\frac{\partial \varphi(x)}{\partial n}\right| d S+\int_{\Gamma^{\delta, m}} d(x)^{3 / 2}|f(x)| d x\right]
\end{aligned}
$$

Remark 2.2. Remark 2.1 extends to both Theorems 2.3 and 2.4.

Remark 2.3. Maz'ya and Plameneveskii [17] use a different method to derive a maximum principle for a conical region: They first establish such a result in an infinite cylinder, and then, by a local Miranda-Agmon maximum principle (Lemma 10.1 in [17]), for a polycylinder. Finally, they map a conical region into a polycylinder. Our approach to derive a maximum principle with integral norms (e. g. Theorem 2.2) is much simpler, and our local MirandaAgmon lemma (which is sharper than Lemma 10.1 in [17]) then yields pointwise estimates for $u, \nabla u$ as in $(2.19),(2.20)$. We note that the Maz'ya-Plameneveskii estimates are in weighted sup norms, similar to $(2.19),(2.20)$; however, for our purposes, the integral estimates will be more convenient. It is also important to note that whereas the conical region in [17] is assumed to have $C^{4}$ boundary, our method allows weaker regularity on the parts of the boundary with zero Dirichlet data. Thus in the case of Theorems 2.3, 2.4, our method requires only that the curve $x_{2}=f\left(x_{1}\right)$ is in $C^{1+\alpha}$.

\section{The stress intensity factors}

Throughout sections $3-6$ we assume that

$$
\begin{aligned}
& \varphi \in H^{2}\left(B_{1} \backslash \Gamma\right), \\
& \Delta^{2} \varphi=0 \quad \text { in } B_{1} \backslash \Gamma, \\
& \varphi=\frac{\partial \varphi}{\partial n}=0 \quad \text { from both sides of } \Gamma,
\end{aligned}
$$


where $B_{1}$ is the unit disc $\left\{x_{1}^{2}+x_{2}^{2}<1\right\}$ and

$$
\Gamma=\left\{x_{2}=f\left(x_{1}\right),-x_{*} \leqslant x_{1} \leqslant 0\right\}
$$

is a curve contained in $B_{1}$ except for its end point $\left(-x_{*}, f\left(-x_{*}\right)\right)$. We also assume that

$$
f(0)=0, \quad f^{\prime}(0)=0 .
$$

In this section and in section 4 , we also assume that $f \in C^{1+\alpha}$, whereas in section 5 we shall require that $f \in C^{2+\alpha}$.

Theorem 3.1 If $f \in C^{1+\alpha}\left[-x_{*}, 0\right]$, then (1.13) holds for any $\eta>0$ such that $\alpha+\eta>1 / 2$.

We shall first prove a weaker result:

$$
\varphi(x)=A_{1} r^{3 / 2} B_{1}(\theta)+A_{2} r^{3 / 2} B_{2}(\theta)+O\left(r^{3 / 2+\lambda}\right) \text { for some } \lambda>0 .
$$

Proof of (3.6). Define

$$
\psi_{\varepsilon}(x)=\frac{\varphi(\varepsilon x)}{\varepsilon^{3 / 2}}, \quad|x|<\frac{1}{\varepsilon}
$$

then

$$
\left|\psi_{\varepsilon}(x)\right| \leqslant C|x|^{3 / 2} \text { for }|x|<\frac{1}{\varepsilon} .
$$

Under this change of variables, $\Gamma$ is changed to

$$
\Gamma_{\varepsilon}: \quad x_{2}=f_{\varepsilon}\left(x_{1}\right) \equiv \frac{1}{\varepsilon} f\left(\varepsilon x_{1}\right),
$$

where $f_{\varepsilon} \in C^{1+\alpha}$, and

$$
f_{\varepsilon}(0)=f_{\varepsilon}^{\prime}(0)=0, \quad\left\|f_{\varepsilon}\right\|_{C^{1+\alpha}\left(-1 \leqslant x_{1} \leqslant 0\right)} \leqslant C \varepsilon^{\alpha} .
$$

Let $G=G_{\varepsilon}$ be a function defined on $\Omega_{2 \pi}\left(\Omega_{\omega}\right.$ is defined in the previous section; here we take $\omega=2 \pi)$ as follows:

$$
\begin{aligned}
& G \in H^{2}\left(\Omega_{2 \pi}\right), \\
& \Delta^{2} G=0 \text { in } \Omega_{2 \pi}, \\
& G=\frac{\partial G}{\partial n}=0 \quad \text { on }\{\theta= \pm \pi\}, \\
& G=\psi_{\varepsilon}, \quad \frac{\partial G}{\partial n}=\frac{\partial \psi}{\partial n} \quad \text { on rest of } \partial \Omega_{2 \pi} .
\end{aligned}
$$

Setting $\omega=2 \pi-2 C \varepsilon^{\alpha}$, we want to apply the Theorem 2.2 to $\psi_{\varepsilon}-G$ in the domain $\Omega_{\omega}$.

For $|x| \leqslant 1 / 2, G$ has an expansion (see $\S 1$ )

$$
G=\sum_{k=1}^{\infty} r^{k / 2+1} \widetilde{B}_{k}(\theta) \quad(r>0,-\pi<\theta<\pi)
$$


where both $\widetilde{B}_{k}$ and their derivatives are bounded. It follows that

$$
\begin{aligned}
& \left.|G|\right|_{|\theta|=\pi-C \varepsilon^{\alpha}} \leqslant C \varepsilon^{2 \alpha}|x|^{3 / 2}, \\
& \left.\left|G_{n}\right|\right|_{|\theta|=\pi-C \varepsilon^{\alpha}} \leqslant C \varepsilon^{\alpha}|x|^{1 / 2} .
\end{aligned}
$$

Since $\psi \in C^{3 / 2}$ by [14], similar estimates are also valid for $\psi$ :

$$
\begin{aligned}
& \left.|\psi|\right|_{|\theta|=\pi-C \varepsilon^{\alpha}} \leqslant C \varepsilon^{3 \alpha / 2}|x|^{3 / 2}, \\
& \left.|\nabla \psi|\right|_{|\theta|=\pi-C \varepsilon^{\alpha}} \leqslant C \varepsilon^{\alpha / 2}|x|^{1 / 2} .
\end{aligned}
$$

It is also clear that a similar estimate is valid for $\psi_{\varepsilon}-G$ on two connecting small smooth arcs near $\left\{|\theta|=\pi-C \varepsilon^{\alpha},|x|=1\right\}$.

By Theorem 2.2, for any $p>2$,

$$
\left\|\psi_{\varepsilon}-G_{\varepsilon}\right\|_{L^{p}\left(B_{1} \cap\left\{-\pi+C \varepsilon^{\alpha}<\theta<\pi-C \varepsilon^{\alpha}\right\}\right)} \leqslant C_{p} \varepsilon^{\alpha / 2} .
$$

Since also

$$
\left|\psi_{\varepsilon}-G_{\varepsilon}\right| \leqslant\left|\psi_{\varepsilon}\right|+\left|G_{\varepsilon}\right| \leqslant C\left|f_{\varepsilon}\right|^{3 / 2}+C \varepsilon^{2 \alpha}|x|^{3 / 2} \leqslant C \varepsilon^{\alpha / 2}|x|^{3 / 2}
$$

in $B_{1} \cap\left\{|\theta \pm \pi| \leqslant C \varepsilon^{\alpha}\right\}$, we get

$$
\left\|\psi_{\varepsilon}-G_{\varepsilon}\right\|_{L^{p}\left(B_{1}\right)} \leqslant C_{p} \varepsilon^{\alpha / 2} .
$$

Notice that $\left|\psi_{\varepsilon}-G_{\varepsilon}\right|$ is uniformly in $C^{3 / 2}$. By interpolation, for any $\beta<\alpha / 2$, we can take $p$ large enough such that

$$
\left|\psi_{\varepsilon}(x)-G_{\varepsilon}(x)\right| \leqslant C \varepsilon^{\beta} \quad \text { for }|x| \leqslant 1 .
$$

Rewriting this in terms of the original variables, we have

$$
\left|\frac{\varphi(x)}{\varepsilon^{3 / 2}}-G_{\varepsilon}\left(\frac{x}{\varepsilon}\right)\right| \leqslant C \varepsilon^{\beta} \quad \text { for }|x| \leqslant \varepsilon .
$$

By (3.13),

$$
G_{\varepsilon}(x)=r^{3 / 2} B_{\varepsilon}(\theta)+O\left(r^{2}\right) \quad \text { for } r=\sqrt{x_{1}^{2}+x_{2}^{2}} \leqslant \frac{1}{2},
$$

where $O\left(r^{2}\right)$ means a term which is bounded by $C r^{2}$ with the constant $C$ independent of $\varepsilon$. Thus

$$
\left|\varphi(x)-r^{3 / 2} B_{\varepsilon}(\theta)\right| \leqslant C \varepsilon^{3 / 2+\beta}+C \varepsilon^{3 / 2}\left(\frac{r}{\varepsilon}\right)^{2} \quad \text { for } r \leqslant \frac{\varepsilon}{2} .
$$

Hence

$$
\left|\frac{\varphi(x)}{r^{3 / 2}}-B_{\varepsilon}(\theta)\right| \leqslant C \varepsilon^{\beta}\left(\frac{\varepsilon}{r}\right)^{3 / 2}+C\left(\frac{r}{\varepsilon}\right)^{1 / 2} \quad \text { for } r \leqslant \frac{\varepsilon}{2} .
$$

Setting $\eta=\beta / 2$, we get

$$
\left|\frac{\varphi(x)}{r^{3 / 2}}-B_{\varepsilon}(\theta)\right| \leqslant C \varepsilon^{\beta}\left(\varepsilon^{-\eta}\right)^{3 / 2}+C \varepsilon^{\eta / 2} \leqslant C \varepsilon^{\beta / 4} \quad \text { for } \varepsilon^{\eta+1} \leqslant r \leqslant 2^{\eta+1} \varepsilon^{\eta+1} .
$$


Now take $\varepsilon_{j}=2^{-j}, r=\varepsilon_{j+1}^{\eta+1}$. Then

$$
\begin{aligned}
\left|B_{\varepsilon_{j}}(\theta)-B_{\varepsilon_{j+1}}(\theta)\right| & \leqslant\left|\frac{\varphi(x)}{r^{3 / 2}}-B_{\varepsilon_{j}}(\theta)\right|+\left|B_{\varepsilon_{j+1}}(\theta)-\frac{\varphi(x)}{r^{3 / 2}}\right| \\
& \leqslant C 2^{-j \beta / 4}=C \varepsilon_{j}^{\beta / 4} .
\end{aligned}
$$

It follows that the series

$$
\sum_{j}\left|B_{\varepsilon_{j}}(\theta)-B_{\varepsilon_{j+1}}(\theta)\right|
$$

is convergent, and

$$
\sum_{j \geqslant k}\left|B_{\varepsilon_{j}}(\theta)-B_{\varepsilon_{j+1}}(\theta)\right| \leqslant C \varepsilon_{k}^{\beta / 4}
$$

Setting

$$
B(\theta)=\lim _{\varepsilon_{j} \rightarrow 0} B_{\varepsilon_{j}}(\theta),
$$

we then have

$$
\left|B(\theta)-B_{\varepsilon_{j}}(\theta)\right| \leqslant C \varepsilon_{j}^{\beta / 4},
$$

so that, by (3.19),

$$
\left|\frac{\varphi(x)}{r^{3 / 2}}-\mathbf{A} \cdot \mathbf{B}(\theta)\right| \leqslant C r^{\beta /[4(\eta+1)]},
$$

where

$$
\begin{aligned}
& \mathbf{A} \cdot \mathbf{B}(\theta)=A_{1} B_{1}(\theta)+A_{2} B_{2}(\theta), \\
& \mathbf{A}=\left(A_{1}, A_{2}\right), \quad \mathbf{B}(\theta)=\left(B_{1}(\theta), B_{2}(\theta)\right),
\end{aligned}
$$

and $B_{1}(\theta), B_{2}(\theta)$ are defined in (1.12). This completes the proof of (3.6).

Let $C_{1}, C_{2}$ be positive constants such that

$$
\|\varphi\|_{H^{2}\left(B_{1} \backslash \Gamma\right)} \leqslant C_{1}, \quad\|f\|_{C^{1+\alpha}} \leqslant C_{2}, \quad 0<\alpha<\frac{1}{2} .
$$

In order to complete the proof of Theorem 3.1, it suffices to prove:

Lemma 3.2 For any $\mu \in\left(0, \min \left(\alpha, \frac{1}{2}\right)\right)$ there exists a constant $C=C_{\mu}$ depending only on $C_{1}, C_{2}$ such that

$$
\left.|\varphi(x)-| x\right|^{3 / 2}\left(A_{1} B_{1}(\theta)+A_{2} B_{2}(\theta)\right) \mid \leqslant C r^{3 / 2+\mu} \quad \text { in } B_{1}
$$

Proof. It is sufficient to show that (3.24) holds for all $r<\frac{1}{2}$. Setting

$$
w(x)=\varphi(x)-A_{1} r^{3 / 2} B_{1}(\theta)-A_{2} r^{3 / 2} B_{2}(\theta),
$$

we have, by (3.6),

$$
|w(x)| \leqslant C r^{3 / 2+\lambda}, \quad \text { for some } 0<\lambda<\alpha .
$$


The proof of (3.25) shows that $C$ depends only on $C_{1}, C_{2}$. If $\mu \leqslant \lambda$, then there is nothing to prove. So we may assume that $\mu>\lambda$; consequently, for any $0<\delta<1$,

$$
\frac{|w(x)|}{r^{3 / 2}\left(r^{\mu}+\delta^{\mu-\lambda} r^{\lambda}\right)}<C
$$

for all $|x|<1$. If $C$ can be chosen to be independent of $\delta$, as well as of $C_{1}, C_{2}$, then (3.24) follows by taking $\delta \rightarrow 0$ in (3.26). So it suffices to show that if such a $C$ does not exist then we get a contradiction.

Assuming that such a $C$ does not exists, there exist sequences $f_{n}, w_{n}, \delta_{n}$ and $x_{n}$ such that

$$
C_{n}=\sup _{|x|<1} \frac{\left|w_{n}(x)\right|}{r^{3 / 2}\left(r^{\mu}+\delta_{n}^{\mu-\lambda} r^{\lambda}\right)}=\frac{\left|w_{n}\left(x_{n}\right)\right|}{R_{n}^{3 / 2}\left(R_{n}^{\mu}+\delta_{n}^{\mu-\lambda} R_{n}^{\lambda}\right)} \rightarrow \infty
$$

if $n \rightarrow \infty$, where $R_{n}=\left|x_{n}\right|<1$. In view of (3.25), we must then have

$$
\delta_{n} \rightarrow 0, \quad R_{n} \rightarrow 0
$$

Introduce a function $G_{n}(\xi)$ by

$$
w_{n}(x)=C_{n} R_{n}^{3 / 2}\left(R_{n}^{\mu}+\delta_{n}^{\mu-\lambda} R_{n}^{\lambda}\right) G_{n}(\xi), \quad x=R_{n} \xi
$$

Then

$$
G_{n}\left(\xi_{n}\right)=1 \quad \text { where } \xi_{n}=\frac{x_{n}}{R_{n}}
$$

and

$$
\begin{aligned}
\left|G_{n}(\xi)\right| & \leqslant \frac{|x|^{3 / 2}\left(|x|^{\mu}+\delta_{n}^{\mu-\lambda}|x|^{\lambda}\right)}{R_{n}^{3 / 2}\left(R_{n}^{\mu}+\delta_{n}^{\mu-\lambda} R_{n}^{\lambda}\right)} \\
& \leqslant|\xi|^{3 / 2} \frac{R_{n}^{\mu-\lambda}|\xi|^{\mu}+\delta_{n}^{\mu-\lambda}|\xi|^{\lambda}}{R_{n}^{\mu-\lambda}+\delta_{n}^{\mu-\lambda}} \\
& \leqslant|\xi|^{3 / 2}\left(|\xi|^{\mu}+|\xi|^{\lambda}\right) .
\end{aligned}
$$

As $n \rightarrow \infty$, the curves $\Gamma_{n}$, defined by $\xi_{2}=f_{n}\left(\xi_{1}\right)$, converge in the $\xi$-plane to the ray

$$
S_{0}=\left\{\left(\xi_{1}, 0\right) ;-\infty<\xi_{1}<0\right\}
$$

and, for a subsequence,

$$
G_{n}(\xi) \rightarrow G(\xi)
$$

uniformly in compact subsets of $\mathbb{R}^{2} \subset \bar{S}_{0}$, and

$$
\begin{aligned}
& \Delta^{2} G=0 \quad \text { in } \mathbb{R}^{2} \backslash \bar{S}_{0}, \\
& G=\frac{\partial G}{\partial \nu}=0 \quad \text { from both sides of } S_{0}, \\
& |G(\xi)| \leqslant|\xi|^{3 / 2}\left(|\xi|^{\mu}+|\xi|^{\lambda}\right) \quad \text { in } \mathbb{R}^{2} \quad\left(0<\lambda<\mu<\min \left(\alpha, \frac{1}{2}\right)\right) .
\end{aligned}
$$


To prove (3.30), we actually need to use sub-Schauder boundary estimates (cf. $\S 9$ ). We first apply Lemma 9.2 to the function $u(x)=\varepsilon^{-3 / 2} \varphi_{n}(\varepsilon x)$ in a ring $1 / 2<|x|<2$ to obtain, for any $\eta \in(0,1)$,

$$
\frac{\left|\varphi_{n}(x)\right|}{|x|^{3 / 2}} \leqslant C\left(\frac{d(x)}{|x|}\right)^{1+\eta}, \quad \frac{\left|\nabla \varphi_{n}(x)\right|}{|x|^{1 / 2}} \leqslant C\left(\frac{d(x)}{|x|}\right)^{\eta},
$$

where $d(x)$ is the distance to the $\Gamma_{n}$. Similar estimates are valid for $|x|^{3 / 2}\left[A_{1} B_{1}(\theta)+A_{2} B_{2}(\theta)\right]$ with $d(x)$ replaced by $\left|x_{2}\right|$. It follows that

$$
\frac{\left|w_{n}(x)\right|}{|x|^{3 / 2}} \leqslant C\left(\frac{d(x)+\left|x_{2}\right|}{|x|}\right)^{1+\eta}, \quad \frac{\left|\nabla w_{n}(x)\right|}{|x|^{1 / 2}} \leqslant C\left(\frac{d(x)+\left|x_{2}\right|}{|x|}\right)^{\eta} .
$$

Since $\mu<\alpha$, we can choose $\eta$ sufficiently close to 1 so that $\alpha \eta>\mu$. Rewriting (3.33) in terms of $\xi$, we then have,

$$
\begin{aligned}
& \left|G_{n}(\xi)\right| \leqslant C_{K} \frac{R_{n}^{3 / 2} R_{n}^{\alpha(1+\eta)}|\xi|^{3 / 2}}{C_{n} R_{n}^{3 / 2}\left(R_{n}^{\mu}+\delta_{n}^{\mu-\lambda} R_{n}^{\lambda}\right)} \leqslant \frac{C_{K}}{C_{n}}|\xi|^{3 / 2} \quad \text { for } \xi \in \mathcal{C}_{K}, \\
& \left|\nabla_{\xi} G_{n}(\xi)\right| \leqslant C_{K} \frac{R_{n}^{1 / 2} R_{n}^{\alpha \eta}|\xi|^{1 / 2}}{C_{n} R_{n}^{1 / 2}\left(R_{n}^{\mu}+\delta_{n}^{\mu-\lambda} R_{n}^{\lambda}\right)} \leqslant \frac{C_{K}}{C_{n}}|\xi|^{1 / 2} \quad \text { for } \xi \in \mathcal{C}_{K},
\end{aligned}
$$

where $\mathcal{C}_{K}=\left\{-K \leqslant \xi_{1} \leqslant 0,\left|\xi_{2}\right| \leqslant-C R_{n}^{\alpha} \xi_{1}\right\}$ (the constant $C$ is from (3.10)). Similarly

$$
\left[\nabla_{\xi} G_{n}\right]_{C^{\beta}\left(\mathcal{C}_{K}\right)} \leqslant \frac{R_{n}^{1 / 2-\beta} R_{n}^{\alpha(\eta-\beta)}}{C_{n} R_{n}^{1 / 2-\beta}\left(R_{n}^{\mu}+\delta_{n}^{\mu-\lambda} R_{n}^{\lambda}\right)} \leqslant \frac{C_{K}}{C_{n}}
$$

provided $\beta$ is taken to be small enough. With these estimates, we can now apply Theorem 9.3 (ii) to $G_{n}(\xi)$ with the boundary $S$ given by $\left|\xi_{2}\right|=-C R_{n}^{\alpha} \xi_{1}$ to obtain $(3.30)$. We also have uniform convergence up to the boundary in (3.28), so that if $\xi_{n} \rightarrow e=\left(e_{1}, e_{2}\right)$, then

$$
|G(e)|=1
$$

We now invoke a Liouville theorem (Lemma 3.3 below) to deduce from (3.29)-(3.31) that $G(\xi) \equiv 0$, which is a contradiction to $(3.37)$.

Lemma 3.3 If $G$ is a function satisfying (3.29)-(3.31), then $G \equiv 0$.

Proof. From the expansion

$$
G(\xi)=\sum_{k=1}^{\infty} r^{k / 2+1} B_{k}(\theta) \quad(r=|\xi|)
$$

near $\xi=0$ and (3.31) (with $\lambda>0$ ) it follows that $B_{1}=0$. Recalling that

$$
r^{2} B_{2}(\theta)=c \xi_{2}^{2}, \quad c \text { constant }
$$


we introduce the function

$$
H(\xi)=G(\xi)-c \xi_{2}^{2}
$$

Then

$$
|H(\xi)| \leqslant C|\xi|^{5 / 2} \text { near } \xi=0
$$

The function

$$
\Phi(\xi)=\frac{\partial H}{\partial \xi_{1}}=\frac{\partial G}{\partial \xi_{1}}
$$

is a biharmonic in $\mathbb{R}^{2} \backslash S_{0}$ and satisfies the same boundary conditions as in (3.30). Since $\Phi$ has an expansion similar to (3.38), we deduce that

$$
\left|\nabla^{j} \Phi(\xi)\right| \leqslant C|\xi|^{5 / 2-1-j} \text { near } \xi=0
$$

Next, for $|\xi|$ large, we have

$$
|G(\xi)| \leqslant 2|\xi|^{3 / 2+\mu}
$$

By scaling and applying elliptic boundary and interior estimates we deduce that

$$
\left|\nabla^{j} \Phi(\xi)\right| \leqslant C|\xi|^{3 / 2+\mu-1-j} \text { for }|\xi| \text { large }
$$

and $0 \leqslant j \leqslant 3$.

Set $D_{R}=\left\{\frac{1}{R}<|\xi|<R\right\} \backslash S_{0}$. By integration by parts,

$$
0=\int_{D_{R}} \Phi \cdot \Delta^{2} \Phi=\int_{D_{R}}|\Delta \Phi|^{2}-I_{R},
$$

where $I_{R}$ is a linear combination of integrals

$$
\begin{aligned}
& A_{R, j}=\int_{\{|\xi|=1 / R\} \backslash S_{0}} D^{j} \Phi \cdot D^{3-j} \Phi \\
& B_{R, j}=\int_{\{|\xi|=R\} \backslash S_{0}} D^{j} \Phi \cdot D^{3-j} \Phi .
\end{aligned}
$$

In view of $(3.39),(3.40)$, we clearly have

$$
\left|A_{R, j}\right| \leqslant \frac{C}{R}, \quad\left|B_{R, j}\right| \leqslant \frac{C R^{2 \mu}}{R},
$$

and, since $\mu<1 / 2, I_{R} \rightarrow 0$ if $R \rightarrow \infty$. It follows that

$$
\int_{\mathbb{R}^{2} \backslash S_{0}}|\Delta \Phi|^{2}=0
$$

so that $\Delta \Phi \equiv 0$. Since $\Phi=\partial \Phi / \partial \nu=0$ on $S_{0}$, we get $\Phi \equiv 0$. This means that

$$
\frac{\partial}{\partial \xi_{1}}\left(G-c \xi_{2}^{2}\right)=0
$$

and so $G=g\left(\xi_{2}\right), g^{(4)}=\Delta^{2} G=0$. Hence $G$ is a polynomial of degree $\leqslant 3$ in $\xi_{2}$, and by invoking (3.30), (3.31) we finally conclude that $G \equiv 0$. 


\section{A flatness lemma}

Let the assumptions of Theorem 3.1 be satisfied and denote by $d(x)$ the distance from $x$ to $\Gamma$. In this section we investigate the behavior of $\varphi(x)$ as $x$ approaches the tip $O$ while, at the same time, $d(x) /|x|$ tends to zero.

Theorem 4.1 Under the assumptions of Theorem 3.1

$$
\frac{|\varphi(x)|}{|x|^{3 / 2}} \leqslant C\left(|x|^{2 \alpha}+\frac{d^{2}(x)}{|x|^{2}}\right)
$$

where $C$ is a constant depending only on the $C_{1}, C_{2}$ in (3.23).

Remark 4.1. If we apply the sub-Schauder estimates $(\S 9$, Lemma 9.2$)$ to $\varepsilon^{-3 / 2} \varphi(\varepsilon x)$ in $1 / 2<|x|<2$, where $\varphi$ is as in Theorem 3.1, we get

$$
\frac{|\varphi(x)|}{|x|^{3 / 2}} \leqslant C\left(\frac{d(x)}{|x|}\right)^{1+\eta} \quad \forall \eta>0 .
$$

The estimate (4.1) is an improvement of (4.2) when

$$
|x|^{\alpha+\eta^{\prime}}<\frac{d(x)}{|x|} \ll 1 \quad \text { for some } \eta^{\prime}>0
$$

To prove Theorem 4.1, we shall establish a lemma which is of intrinsic interest.

Let

$$
\begin{aligned}
& \Gamma=\left\{x_{2}=f\left(x_{1}\right),-1<x_{1}<1\right\}, \\
& D=\left\{\left(x_{1}, x_{2}\right) ;-1<x_{1}<1,-1<x_{2}<f\left(x_{1}\right)\right\},
\end{aligned}
$$

and let $\gamma$ be a subarc of $\Gamma$ :

$$
\gamma=\left\{x_{2}=f\left(x_{1}\right),-1 / 2<x_{1}<1 / 2\right\}
$$

We assume a bound

$$
\|f\|_{C^{1+\alpha}} \leqslant A \quad(0<\alpha<1, \quad A>0)
$$

and the " $\varepsilon$-flatness" condition:

$$
\left|f\left(x_{1}\right)\right|<\varepsilon \quad(0<\varepsilon<1 / 2) .
$$

Denote by $d(x)$ the distance function

$$
d(x)=\operatorname{dist}(x, \gamma), \quad x \in D
$$


Lemma 4.2 (Flatness Lemma) If $\psi \in H^{2}(D \backslash \Gamma),\|\psi\|_{H^{2}(D \backslash \Gamma)} \leqslant C_{1}$,

$$
\begin{aligned}
& \Delta^{2} \psi=0 \quad \text { in } D, \\
& \psi=\frac{\partial \psi}{\partial \nu}=0 \quad \text { on } \Gamma, \\
& |\psi|<1 \quad \text { in } D,
\end{aligned}
$$

then

$$
|\psi(x)| \leqslant C\left(d^{2}(x)+\varepsilon^{2}\right) \quad \text { in } D
$$

where $C$ is a constant depending only on $A, \alpha$ and $C_{1}$.

Remark 4.2. By sub-Schauder estimates (see Example 2 following Theorem 9.1)

$$
|\psi(x)| \leqslant C d^{2-\eta}(x) \quad \forall \eta>0 ;
$$

(4.8) is an improvement when $d(x) \gg \varepsilon^{1+\eta^{\prime}}$ for some $\eta^{\prime}>0$.

Proof. We assume that (4.8) is not valid and derive a contradiction. If (4.8) is not true then there exist sequences

$$
\Gamma=\Gamma_{n}, \quad \gamma=\gamma_{n}, \quad D=D_{n}, \quad \varepsilon=\varepsilon_{n}, \quad \psi=\psi_{n},
$$

such that, with $d_{n}(x)=\operatorname{dist}\left(x, \gamma_{n}\right)$,

$$
C_{n}=\sup _{D_{n}} \frac{\left|\psi_{n}(x)\right|}{d_{n}^{2}(x)+\varepsilon_{n}^{2}}=\frac{\left|\psi_{n}\left(x_{n}\right)\right|}{d_{n}^{2}\left(x_{n}\right)+\varepsilon_{n}^{2}} \rightarrow \infty \quad \text { if } n \rightarrow \infty,
$$

where $x_{n} \in D_{n}$; we necessarily have

$$
d_{n} \equiv d_{n}\left(x_{n}\right) \rightarrow 0, \quad \varepsilon_{n} \rightarrow 0 \quad \text { if } n \rightarrow \infty
$$

for otherwise the sequence $C_{n}$ will remain bounded (by (4.7)). Denote by $\widetilde{x}_{n}$ the point on $\gamma_{n}$ such that $d_{n}=\left|x_{n}-\widetilde{x}_{n}\right|$.

Introduce functions $G_{n}(\xi)$ by

$$
\psi_{n}(x)=C_{n}\left(d_{n}^{2}+\varepsilon_{n}^{2}\right) G_{n}(\xi) \text { where } x-\widetilde{x}_{n}=d_{n} \xi .
$$

Then

$$
\begin{aligned}
& d_{n}(x)=d_{n} \tilde{d}_{n}(\xi), \\
& G_{n}\left(\xi_{n}\right)=1, \quad \xi_{n}=\frac{x_{n}-\widetilde{x}_{n}}{d_{n}}, \quad \tilde{d}_{n}\left(\xi_{n}\right)=1,
\end{aligned}
$$

where $\widetilde{d}_{n}(\xi)$ is the distance from $\xi$ to the image $\widetilde{\gamma}_{n}$ of $\gamma_{n}$ under the mapping $x-\widetilde{x}_{n}=d_{n} \xi$.

Clearly,

$$
\left|G_{n}(\xi)\right| \leqslant \frac{d_{n}^{2}(x)+\varepsilon_{n}^{2}}{d_{n}^{2}+\varepsilon_{n}^{2}} \leqslant \frac{d_{n}^{2} \widetilde{d}_{n}^{2}(\xi)+\varepsilon_{n}^{2}}{d_{n}^{2}+\varepsilon_{n}^{2}}
$$


It is sufficient to consider the following two cases:

Case (1): $d_{n} / \varepsilon_{n} \rightarrow 0$ if $n \rightarrow \infty$.

Case (2): $d_{n} \geqslant c \varepsilon_{n}$ for some $c>0$ for all large $n$.

In case $(1),(4.11)$ clearly implies that

$$
\left|G_{n}(\xi)\right| \leqslant \widetilde{d}_{n}^{2}(\xi)\left(\frac{d_{n}}{\varepsilon_{n}}\right)^{2}+1,
$$

and in case (2), (4.11) implies that

$$
\left|G_{n}(\xi)\right| \leqslant \frac{d_{n}^{2}(x)}{d_{n}^{2}}+1 \leqslant|\xi|^{2}+1
$$

As $n \rightarrow \infty$, the curves $\widetilde{\gamma}_{n}$ in the $\xi$-plane converges to the line $\left\{\xi_{2}=0\right\}$ and, by $(4.10)$,

$$
G_{n}(\xi) \rightarrow G(\xi)
$$

uniformly in compact subsets of $\left\{\xi_{2}<0\right\}$,

$$
\begin{aligned}
& \Delta^{2} G(\xi)=0 \quad \text { in }\left\{\xi_{2}<0\right\}, \\
& |G(\xi)| \leqslant 1 \quad \text { in }\left\{\xi_{2}<0\right\} \quad \text { in case }(1), \\
& |G(\xi)| \leqslant|\xi|^{2}+1 \quad \text { in }\left\{\xi_{2}<0\right\} \quad \text { in case }(2) .
\end{aligned}
$$

By sub-Schauder estimates (Lemma 9.2) applied to $G_{n}(\xi)$ we deduce that

$$
G=\frac{\partial G}{\partial \nu}=0 \quad \text { on }\left\{\xi_{2}=0\right\}
$$

We also note that the convergence in $(4.10)$ is uniform near the boundary so that, in particular, if

$$
\xi_{n}=\frac{x_{n}-\tilde{x}_{n}}{d_{n}} \rightarrow e \quad\left(e=\left(e_{1}, e_{2}\right), e_{2} \leqslant 0,|e|=1\right)
$$

then

$$
|G(e)|=\lim \left|G_{n}\left(\xi_{n}\right)\right|=1
$$

By a Liouville theorem (Lemma 4.3 below) we conclude from (4.15)-(4.17), (4.18) and (4.19) that

$$
G(\xi)=K \xi_{2}^{2}, K \neq 0
$$

This contradicts (4.16) (case (1)).

We shall next derive a contradiction to (4.20) (case (2)). The proof depends on sharp estimates on the $\psi_{n}(x)$. Since,

$$
\left|\psi_{n}(x)\right| \leqslant C_{n}\left(d_{n}^{2}(x)+\varepsilon_{n}^{2}\right) \quad \text { in } D_{n}
$$

the flatness condition implies that

$$
\left|\psi_{n}(x)\right| \leqslant C C_{n} \varepsilon_{n}^{2} \quad \text { in }\left\{\left|x_{1}\right|<\frac{1}{2},-4 \varepsilon_{n}<x_{2}<-2 \varepsilon_{n}\right\} .
$$


By interior elliptic estimates we then also have

$$
\left|\nabla \psi_{n}(x)\right| \leqslant C C_{n} \varepsilon_{n} \quad \text { on }\left\{\left|x_{1}\right|<\frac{1}{4}, x_{2}=-3 \varepsilon_{n}\right\} .
$$

We need to construct an auxiliary function. For this purpose we first consider the problem

$$
\begin{aligned}
& \Delta^{2} \varphi=0 \quad \text { in }\left\{x_{2}>0\right\}, \\
& \varphi\left(x_{1}, 0\right)=f_{0}\left(x_{1}\right), \\
& \frac{\partial}{\partial x_{2}} \varphi\left(x_{1}, 0\right)=g_{0}\left(x_{1}\right),
\end{aligned}
$$

where $f_{0} \in C_{l o c}^{1+\alpha}, g_{0} \in C_{l o c}^{\alpha}$.

If $f_{0}, g_{0}$ are uniformly bounded, then we can write a solution in the form

$$
\varphi=P\left(f_{0}\right)+x_{2}\left[P\left(g_{0}\right)-\frac{\partial}{\partial x_{2}} P\left(f_{0}\right)\right]
$$

where $P$ is the Poisson kernel:

$$
P(h)(x)=\frac{1}{\pi} \int_{-\infty}^{\infty} \frac{x_{2}}{\left(x_{1}-\xi_{1}\right)^{2}+x_{2}^{2}} h\left(\xi_{1}\right) d \xi_{1} .
$$

Noting that

$$
\frac{\partial}{\partial x_{2}} P\left(f_{0}\right)=\frac{1}{\pi} \int_{-\infty}^{\infty} \frac{\left(x_{1}-\xi_{1}\right)^{2}-x_{2}^{2}}{\left[\left(x_{1}-\xi_{1}\right)^{2}+x_{2}^{2}\right]^{2}} f_{0}\left(\xi_{1}\right) d \xi_{1},
$$

we find that

$$
|\varphi(x)| \leqslant C\left|f_{0}\right|_{L^{\infty}}+C\left|x_{2}\right|\left(\left|g_{0}\right|_{L^{\infty}}+\left|f_{0}\right|_{L^{\infty}}\right) .
$$

Let $\chi\left(x_{1}\right)$ be a $C^{2}$ function such that $\chi\left(x_{1}\right)=1$ if $\left|x_{1}\right|<1 / 8, \chi\left(x_{1}\right)=0$ if $\left|x_{1}\right|>1 / 4$. Introduce the functions

$$
f_{0}=\chi_{\psi_{n}}, g_{0}=\frac{\partial}{\partial x_{2}}\left(\chi_{\psi_{n}}\right) \quad \text { on } x_{2}=-3 \varepsilon_{n}
$$

and denote by $\Phi_{n}$ the biharmonic function in $\left\{x_{2}<-3 \varepsilon_{n}\right\}$ with Dirichlet data $f_{0}, g_{0}$ on $x_{2}=-3 \varepsilon_{n}$. Using a representation similar to (4.23) (with $\left(x_{1}, x_{2}\right)$ replaced by $\left(x_{1},-x_{2}-3 \varepsilon_{n}\right)$ ) we get, from (4.24) and (4.21), (4.22), the estimate

$$
\left|\Phi_{n}(x)\right| \leqslant C C_{n}\left(\varepsilon_{n}^{2}+\left|x_{2}\right| \varepsilon_{n}\right) \quad \text { if } x_{2} \leqslant-3 \varepsilon_{n} .
$$

Consider the function

$$
\zeta_{n}=\psi_{n}-\Phi_{n} \quad \text { in } \Omega_{n}=\left\{\left|x_{1}\right|<\frac{1}{8},-1<x_{2}<-3 \varepsilon_{n}\right\} .
$$

It satisfies

$$
\begin{aligned}
& \Delta^{2} \zeta_{n}=0 \quad \text { in } \Omega_{n}, \\
& \zeta_{n}=\frac{\partial \zeta_{n}}{\partial x_{2}}=0 \quad \text { on } x_{2}=-3 \varepsilon_{n},\left|x_{1}\right|<\frac{1}{8},
\end{aligned}
$$


and

$$
\left|\zeta_{n}(x)\right| \leqslant 1+C C_{n} \varepsilon_{n} \equiv K_{n} \quad \text { in } \Omega_{n}
$$

by (4.25) and (4.7).

By standard elliptic regularity

$$
\frac{1}{K_{n}}\left|\zeta_{n}\left(x_{1},-3 \varepsilon_{n}-M d_{n}\right)\right| \leqslant C\left(M d_{n}\right)^{2} \quad \text { if }\left|x_{1}\right|<\frac{1}{16}
$$

for any $M>0$, and we shall later choose $M \gg 1$ (but $M d_{n}+3 \varepsilon_{n}<1$ ). Combining this with (4.25), we get

$$
\left|\psi_{n}\left(0,-3 \varepsilon_{n}-M d_{n}\right)\right| \leqslant C C_{n}\left(\varepsilon_{n}^{2}+d_{n} M \varepsilon_{n}\right)+\left(1+C C_{n} \varepsilon_{n}\right) C\left(M d_{n}\right)^{2} .
$$

On the other hand, by (4.10), (4.14), (4.20),

$$
\psi_{n}\left(0,-3 \varepsilon_{n}-M d_{n}\right) \geqslant C_{n} d_{n}^{2} G_{n}\left(\widehat{\xi}_{n}\right) \sim C_{n} d_{n}^{2} G\left(\widehat{\xi}_{n}\right)=K C_{n} d_{n}^{2}\left|\widehat{\xi}_{n, 2}\right|^{2}
$$

where

$$
\left(0,-\varepsilon_{n}-M d_{n}\right)-\widetilde{x}_{n}=d_{n} \widehat{\xi}_{n}=d_{n}\left(\widehat{\xi}_{n, 1}, \widehat{\xi}_{n, 2}\right)
$$

and $\left|\widehat{\xi}_{n, 2}\right| \sim M$ if $M \gg 1$. Comparing this with (4.26) we get

$$
K C_{n} d_{n}^{2} M^{2} \leqslant C C_{n} M \varepsilon_{n}^{2}+C\left(1+C C_{n} \varepsilon_{n}\right) M^{2} d_{n}^{2} .
$$

Since $d_{n} \geqslant c \varepsilon_{n}(c>0)$, choosing $M$ to be large enough (say $K M>2 C$ ), we get a contradiction as we let $n \rightarrow \infty$.

Lemma 4.3 If $G(\xi)$ is a function satisfying (4.15),(4.17), (4.18), and if $|G(\xi)| \leqslant C\left(1+|\xi|^{2}\right)$ in $\left\{\xi_{2}<0\right\}$, then

$$
G(\xi) \equiv K \xi_{2}^{2}
$$

where $K$ is a constant.

Proof. The biharmonic function

$$
H(\xi)=\frac{\partial^{2}}{\partial \xi_{1}^{2}} G(\xi)
$$

satisfies the same boundary condition as $G$, and

$$
\left|\nabla^{j} H(\xi)\right| \leqslant C|\xi|^{-j} \quad \text { if }|\xi| \text { is large. }
$$

By integration by parts,

$$
\int_{\left\{|\xi|<R, \xi_{2}<0\right\}} H \Delta^{2} H=\int_{\left\{|\xi|<R, \xi_{2}<0\right\}}(\Delta H)^{2}+I_{R}
$$

where $I_{R} \rightarrow 0$ if $R \rightarrow \infty$ (cf. the proof of Lemma 3.3). If follows that $\Delta H \equiv 0$ and, by unique continuation, $H \equiv 0$. Hence $G=g_{1}\left(\xi_{2}\right)+g_{2}\left(\xi_{2}\right) \xi_{1}$ and this implies (4.27).

Proof of Theorem 4.1. Applying the flatness lemma to $\varepsilon^{-3 / 2} \varphi(\varepsilon x)$ for $1 / 2<|x|<2$ and arbitrarily small $\varepsilon$, the assertion (4.1) easily follows. 


\section{Higher order expansion}

In this section we assume that $f \in C^{2+\alpha}$ and obtain higher order expansion of $\varphi$.

Theorem 5.1 Let $\varphi$ be a solution of (3.1)-(3.5) and assume that $f \in C^{2+\alpha}\left[-x_{*}, 0\right]$ for some $\alpha>0$. Then for any $0<\eta<1 / 2$ such that $\alpha+\eta>1 / 2$, the expansion (1.14) holds.

The assumptions of Theorem 5.1 imply that

$$
\|\varphi\|_{H^{2}\left(B_{1} \backslash \Gamma\right)} \leqslant C_{1}, \quad\|f\|_{C^{2+\alpha}} \leqslant C_{2} .
$$

For clarity we shall first prove a special case:

Lemma 5.2 Under the assumptions of Theorem 5.1, for any $\eta \in(0,1 / 2)$ there exists a constant $C$ depending only on $C_{1}, C_{2}$ and $\eta$ such that

$$
\left|\varphi(x)-r^{3 / 2}\left[A_{1} B_{1}(\theta)+A_{2} B_{2}(\theta)\right]-A_{3} r^{2} B_{3}(\theta)\right| \leqslant C r^{2+\eta} .
$$

In the sequel we shall need the following interpolation inequality:

$$
\|\nabla u\|_{L^{\infty}} \leqslant C\left(\|u\|_{L^{\infty}}^{\sigma /(1+\sigma)}\|\nabla u\|_{C^{\sigma}}^{1 /(1+\sigma)}+\|u\|_{L^{\infty}}\right) \quad(0<\sigma<1)
$$

where the norms are taken in a bounded domain $\Omega$. It suffices to prove (5.2) in dimension 1. For any $x \in \Omega$, let $y, z$ belong to $\Omega$ such that

$$
|y-x|=\varepsilon, \quad|z-x|=\varepsilon
$$

Then

$$
\frac{u(y)-u(x)}{y-x}=u_{x}(\tilde{x})
$$

and

$$
u_{x}(z)=u_{x}(z)-u_{x}(\tilde{x})+\frac{u(y)-u(x)}{y-x}
$$

Hence

$$
\left\|u_{x}\right\|_{L^{\infty}} \leqslant\left\|u_{x}\right\|_{C^{\sigma} \varepsilon^{\sigma}}+\frac{2}{\varepsilon}\|u\|_{L^{\infty}}
$$

and choosing

$$
\varepsilon^{\sigma+1}=\|u\|_{L^{\infty}} /\left(\left\|u_{x}\right\|_{C^{\sigma}}+\|u\|_{L^{\infty}}\right)
$$

yields the assertion.

In the sequel we shall also use the interpolation inequality

$$
\|\zeta\|_{C^{\theta}} \leqslant C\left(\|\zeta\|_{L^{\infty}}^{(\beta-\theta) / \beta}[\zeta]_{C^{\beta}}^{\theta / \beta}+\|\zeta\|_{L^{\infty}}\right) \quad(0<\theta<\beta<1),
$$

which follows from

$$
\frac{|\zeta(x)-\zeta(y)|}{|x-y|^{\theta}}=\frac{|\zeta(x)-\zeta(y)|}{|x-y|^{\beta}}|x-y|^{\beta-\theta} \leqslant[\zeta]_{C^{\beta}} \delta^{\beta-\theta}+\frac{2\|\zeta\|_{L^{\infty}}}{\delta^{\theta}}
$$


by taking $\delta=\left(\|\zeta\|_{L^{\infty}} /\|\zeta\|_{C^{\beta}}\right)^{1 / \beta}$.

Proof of Lemma 5.2. The function

$$
\psi_{\varepsilon}(x)=\frac{1}{\varepsilon^{3 / 2}} \varphi(\varepsilon x)
$$

is biharmonic in $B_{4}(0) \backslash \Gamma_{\varepsilon}$, where

$$
\Gamma_{\varepsilon}: x_{2}=\frac{1}{\varepsilon} f\left(\varepsilon x_{1}\right) \equiv f_{0}\left(x_{1}\right)
$$

is uniformly $C^{2, \alpha}$. By sub-Schauder estimates ( $c f . \S 9$, Theorem 9.3),

$$
\left|D^{2} \psi_{\varepsilon}\right|+\left[D^{2} \psi_{\varepsilon}\right]_{\alpha} \leqslant C \quad \text { for } 1<|x|<2
$$

Rewriting this inequality in terms of $\varphi$, we get

$$
\left|D^{2} \varphi(x)\right| \leqslant C|x|^{-1 / 2}, \quad\left[D^{2} \varphi\right]_{C^{\alpha}[|x|<r<1]} \leqslant C|x|^{-1 / 2-\alpha}
$$

where the constant $C$ is independent of $\varepsilon$. Multiplying by a constant if necessary, we may assume without loss of generality that

$$
\left|\psi_{\varepsilon}\right| \leqslant 1, \quad\left|\nabla \psi_{\varepsilon}\right| \leqslant 1 \quad \text { for }|x|<1,0<\varepsilon<1
$$

Let $\varphi_{0}=\psi_{\varepsilon}$ where $\varepsilon$ will later on be chosen very small but fixed, and denote by $G_{0}$ the solution of

$$
\begin{aligned}
& G \in H^{2}\left(B_{1}(0) \backslash\{\theta= \pm \pi\}\right), \\
& \Delta^{2} G=0 \quad \text { in } B_{1}(0) \backslash\{\theta= \pm \pi\}, \\
& G=\frac{\partial G}{\partial n}=0 \text { on }\{\theta= \pm \pi\}, \\
& G=\varphi_{0}, \quad \frac{\partial G}{\partial n}=\frac{\partial \varphi_{0}}{\partial n} \quad \text { on rest of } \partial\left(B_{1}(0) \backslash\{\theta= \pm \pi\}\right) .
\end{aligned}
$$

It is clear that

$$
\left|f_{0}^{\prime \prime}\left(x_{1}\right)\right|=\varepsilon\left|f^{\prime \prime}\left(\varepsilon x_{1}\right)\right| \leqslant \varepsilon,
$$

and

$$
\left|f_{0}\left(x_{1}\right)\right| \leqslant \frac{1}{2} \varepsilon\left|x_{1}\right|^{2}
$$

Hence, using (5.4),

$$
\begin{aligned}
& \left|\varphi_{0}\right|+\left|G_{0}\right| \leqslant C|x|^{-1 / 2}\left(\varepsilon\left|x_{1}\right|\right)^{2} \leqslant C \varepsilon^{2}|x|^{3 / 2} \quad \text { on }|\theta \pm \pi| \leqslant 2 \varepsilon \\
& \left|\nabla \varphi_{0}\right|+\left|\nabla G_{0}\right| \leqslant C|x|^{-1 / 2}\left(\varepsilon\left|x_{1}\right|\right) \leqslant C \varepsilon|x|^{1 / 2} \quad \text { on }|\theta \pm \pi| \leqslant 2 \varepsilon
\end{aligned}
$$

Applying Theorem 2.2, we get

$$
\left\|\varphi_{0}-G_{0}\right\|_{L^{p}\left(\Omega_{2 \pi-2 \varepsilon}\right)} \leqslant C \varepsilon .
$$


Notice that both $\varphi_{0}$ and $G_{0}$ are uniformly $C^{3 / 2}$. It is clear that if $\sigma<1 / 4-\eta / 2$ (recall that $0<\eta<1 / 2)$, then $\left[\nabla\left(\varphi_{0}-G_{0}\right)\right]_{C^{\sigma}} \leqslant C$ for some universal constant $C$ (Actually, $\varphi_{0}-G_{0}$ is bounded also in the $C^{1 / 2}$ norm in this first step of the iteration; but we use the $C^{\sigma}$ norm for the later iterations). Thus by interpolation, for any $\beta<1$, if we choose $p$ large enough we obtain

$$
\left|\varphi_{0}-G_{0}\right| \leqslant C \varepsilon^{\beta} \quad \text { for }|x|<1
$$

By (5.2) with $u=\varphi_{0}-G_{0}$, we also have

$$
\left|\nabla\left(\varphi_{0}-G_{0}\right)\right| \leqslant C\left|\varphi_{0}-G_{0}\right|^{\sigma /(1+\sigma)}\left[\nabla\left(\varphi_{0}-G_{0}\right)\right]_{C^{\sigma}}^{1 /(1+\sigma)}+C \varepsilon^{\beta} \leqslant C \varepsilon^{\beta \sigma /(1+\sigma)} \quad \text { for }|x|<1 .
$$

Clearly, there is a constant $C$ and $P_{0}(x)=\left[r^{3 / 2} B_{\varepsilon}(\theta)+r^{2} \widetilde{B}_{\varepsilon}(\theta)\right]$ such that

$$
\begin{aligned}
& \left|G_{0}-P_{0}\right| \leqslant C r^{5 / 2} \quad \text { for } r<1 / 2, \\
& \left|\nabla\left(G_{0}-P_{0}\right)\right| \leqslant C r^{3 / 2} \quad \text { for } r<1 / 2 .
\end{aligned}
$$

It follows that

$$
\begin{aligned}
& \left|\varphi_{0}-P_{0}\right| \leqslant C \varepsilon^{\beta}+C r^{5 / 2} \quad \text { for } r<1 / 2, \\
& \left|\nabla\left(\varphi_{0}-P_{0}\right)\right| \leqslant C \varepsilon^{\beta \sigma /(1+\sigma)}+C r^{3 / 2} \quad \text { for } r<1 / 2 .
\end{aligned}
$$

We now fix small constants $\varepsilon, \lambda$ such that

$$
C \varepsilon^{\beta}+C(2 \lambda)^{5 / 2} \leqslant \lambda^{2+\eta}, \quad C \varepsilon^{\beta \sigma /(1+\sigma)}+C(2 \lambda)^{3 / 2} \leqslant \lambda^{1+\eta}
$$

we can actually take $\varepsilon=\lambda^{\gamma}$ for $\gamma$ large enough and any $\lambda$ sufficiently small. Since $\varepsilon$ is now fixed, we shall simply write $P_{0}$ as

$$
P_{0}=r^{3 / 2} B^{0}(\theta)+r^{2} \widetilde{B}^{0}(\theta),
$$

and we then have

$$
\begin{aligned}
& \left|\varphi_{0}-P_{0}\right| \leqslant \lambda^{2+\eta} \quad \text { for }|x|<2 \lambda, \\
& \left|\nabla\left(\varphi_{0}-P_{0}\right)\right| \leqslant \lambda^{1+\eta} \quad \text { for }|x|<2 \lambda .
\end{aligned}
$$

Next, we define

$$
\varphi_{1}=\frac{1}{\lambda^{2+\eta}}\left(\varphi_{0}(\lambda x)-P_{0}(\lambda x)\right) \quad \text { for } r \leqslant 2
$$

Then

$$
\left|\varphi_{1}\right| \leqslant 1, \quad\left|\nabla \varphi_{1}\right| \leqslant 1 \quad \text { for } r \leqslant 2
$$

and, by (5.7), (5.8)

$$
\begin{array}{ll}
\left|\varphi_{1}\right| \leqslant C \lambda^{-2-\eta}|\lambda x|^{-1 / 2}\left(\varepsilon \lambda\left|\lambda x_{1}\right|\right)^{2} \leqslant C \varepsilon^{2} \lambda^{3 / 2-\eta}|x|^{3 / 2} & \text { for }|\theta \pm \pi|<2(\varepsilon \lambda), \\
\left|\nabla \varphi_{1}\right| \leqslant C \lambda^{-1-\eta}|\lambda x|^{-1 / 2}\left(\varepsilon \lambda\left|\lambda x_{1}\right|\right) \leqslant C \varepsilon \lambda^{1 / 2-\eta}|x|^{1 / 2} & \text { for }|\theta \pm \pi|<2(\varepsilon \lambda) .
\end{array}
$$


Finally, since $\left|D^{2} \varphi_{0}\right|+\left|D^{2} G_{0}\right| \leqslant C|x|^{-1 / 2}$,

$$
\left[\nabla \varphi_{1}\right]_{C^{\sigma}(\{|\theta \pm \pi|<2(\varepsilon \lambda)\} \cap\{r<|x|\})} \leqslant C \lambda^{-1-\eta}|\lambda x|^{-1 / 2}\left(\varepsilon \lambda\left|\lambda x_{1}\right|\right)^{1-\sigma} \leqslant C \varepsilon^{1-\sigma}|x|^{1 / 2-\sigma}
$$

since $\sigma<1 / 4-\eta / 2$. We claim that

$$
\left|\varphi_{1}(x)\right| \leqslant|x|^{1+\sigma} \quad \text { if }|x|<2
$$

For clarity of exposition we shall postpone the proof until the end of this section, and in fact, establish a general result (namely Lemma 5.4) for which (5.21) follows as a special case (upon taking $f_{j}\left(x_{1}\right)$ to be parabolic curves $x_{2}=\delta_{j} x_{1}^{2}$, with $-1<\delta_{1}<\delta_{2}<1$; notice that $\varphi_{1}$ is biharmonic outside the thin region enclosed by these two parabolic curves).

In view of (5.21), we can apply Theorem 9.3 (ii) to the function

$$
\frac{\varphi_{1}(\tau x)}{\tau^{1+\sigma}}
$$

which (by (5.21)) is bounded in $1 / 2<|x|<2$ and whose $C^{1+\sigma}$-norm is uniformly bounded in the sector $|\theta \pm \pi| \leqslant 2(\varepsilon \lambda)$ (by (5.19), (5.20)). We then have

$$
\left[\nabla \varphi_{1}\right]_{C^{\sigma}\left(B_{1}\right)} \leqslant C
$$

for some universal constant $C$. We can now proceed as above (with the same $\varepsilon, \lambda$ ) to derive

$$
\begin{aligned}
& \left|\varphi_{1}-P_{1}\right| \leqslant \lambda^{2+\eta} \quad \text { for }|x|<2 \lambda, \\
& \left|\nabla\left(\varphi_{1}-P_{1}\right)\right| \leqslant \lambda^{1+\eta} \quad \text { for }|x|<2 \lambda
\end{aligned}
$$

for some $P_{1}(x)=r^{3 / 2} B^{1}(\theta)+r^{2} \widetilde{B}^{1}(\theta)$.

Proceeding by induction, we define

$$
\begin{aligned}
\varphi_{k+1}(x) & =\frac{1}{\lambda^{2+\eta}}\left(\varphi_{k}(\lambda x)-P_{k}(\lambda x)\right) \\
& =\left(\lambda^{-(2+\eta)}\right)^{k+1} \varphi_{0}\left(\lambda^{k+1} x\right)-\sum_{j=0}^{k}\left(\lambda^{-(2+\eta)}\right)^{j+1} P_{k-j}\left(\lambda^{j+1} x\right) .
\end{aligned}
$$

Clearly,

$$
\begin{aligned}
\left|\left(\lambda^{-(2+\eta)}\right)^{k+1} \varphi_{0}\left(\lambda^{k+1} x\right)\right| & \leqslant C \lambda^{-(2+\eta)(k+1)}\left|\lambda^{k+1} x\right|^{-1 / 2}\left(\varepsilon \lambda^{k+1}\left|\lambda^{k+1} x\right|\right)^{2} \\
& \leqslant C \varepsilon^{2} \lambda^{(3 / 2-\eta) k}|x|^{3 / 2} \quad \text { for }|\theta \pm \pi|<2 \varepsilon \lambda^{k+1}
\end{aligned}
$$

and

$$
\begin{aligned}
\sum_{j=0}^{k}\left(\lambda^{-(2+\eta)}\right)^{j+1}\left|P_{k-j}\left(\lambda^{j+1} x\right)\right| & \leqslant C \sum_{j=0}^{k}\left(\lambda^{-(2+\eta)}\right)^{j+1}\left|\lambda^{j+1} x\right|^{-1 / 2}\left(\varepsilon \lambda^{k+1}\left|\lambda^{j+1} x\right|\right)^{2} \\
& \leqslant C \varepsilon^{2} \lambda^{(3 / 2-\eta) k}|x|^{3 / 2} \quad \text { for }|\theta \pm \pi|<2 \varepsilon \lambda^{k+1}
\end{aligned}
$$


It follows that

$$
\left|\varphi_{k+1}(x)\right| \leqslant C \varepsilon^{2} \lambda^{(3 / 2-\eta)(k+1)}|x|^{3 / 2} \quad \text { for }|\theta \pm \pi|<2 \varepsilon \lambda^{k+1} \text {. }
$$

Similarly,

$$
\begin{aligned}
\left|\nabla\left[\left(\lambda^{-(2+\eta)}\right)^{k+1} \varphi_{0}\left(\lambda^{k+1} x\right)\right]\right| & \leqslant C \lambda^{-(1+\eta)(k+1)}\left|\lambda^{k+1} x\right|^{-1 / 2}\left(\varepsilon \lambda^{k+1}\left|\lambda^{k+1} x\right|\right) \\
& \leqslant C \varepsilon \lambda^{(1 / 2-\eta)(k+1)}|x|^{1 / 2} \quad \text { for }|\theta \pm \pi|<2 \varepsilon \lambda^{k+1}
\end{aligned}
$$

and

$$
\begin{aligned}
\sum_{j=0}^{k}\left(\lambda^{-(2+\eta)}\right)^{j+1}\left|\nabla\left(P_{k-j}\left(\lambda^{j+1} x\right)\right)\right| & \leqslant C \sum_{j=0}^{k}\left(\lambda^{-(1+\eta)}\right)^{j+1}\left|\lambda^{j+1} x\right|^{-1 / 2}\left(\varepsilon \lambda^{k+1}\left|\lambda^{j+1} x\right|\right) \\
& \leqslant C \varepsilon \lambda^{(1 / 2-\eta) k}|x|^{1 / 2} \quad \text { for }|\theta \pm \pi|<2 \varepsilon \lambda^{k+1}
\end{aligned}
$$

It follows that

$$
\left|\nabla \varphi_{k+1}(x)\right| \leqslant C \varepsilon \lambda^{(1 / 2-\eta)(k+1)}|x|^{1 / 2} \quad \text { for }|\theta \pm \pi|<2 \varepsilon \lambda^{k+1}
$$

We can apply the same procedure to deduce that

$$
\left[\nabla \varphi_{k+1}(x)\right]_{C \sigma\left(\left\{|\theta \pm \pi|<2 \varepsilon \lambda^{k+1}\right\} \cap\{r<|x|\}\right)} \leqslant C \varepsilon^{1-\sigma}|x|^{1 / 2-\sigma} .
$$

With the estimates (5.25) and (5.26) and (5.27) at hand (where the constants $C$ are independent of $\varepsilon, \lambda, k)$, the same procedure can be used to deduce that

$$
\begin{aligned}
& \left|\varphi_{k+1}-P_{k+1}(x)\right| \leqslant \lambda^{2+\eta} \quad \text { for }|x| \leqslant 2 \lambda, \\
& \left|\nabla\left(\varphi_{k+1}-P_{k+1}(x)\right)\right| \leqslant \lambda^{1+\eta} \quad \text { for }|x| \leqslant 2 \lambda .
\end{aligned}
$$

We can rewrite the inequality (5.28) in terms of the original variables:

$$
\left|\varphi_{0}(x)-Q_{k}(x)\right| \leqslant\left(\lambda^{k}\right)^{2+\eta} \quad \text { for }|x|<2 \lambda^{k},
$$

where

$$
Q_{k}(x)=\sum_{j=0}^{k}\left(\lambda^{j}\right)^{2+\eta} P_{j}\left(\frac{x}{\lambda^{j}}\right)
$$

Since $P_{j}\left(x / \lambda^{j}\right)$ are bounded by $C\left|x / \lambda^{j}\right|^{2}+C\left|x / \lambda^{j}\right|^{3 / 2}$, the series converges, and we let

$$
Q(x)=\lim _{k \rightarrow \infty} Q_{k}(x)=\sum_{j=0}^{\infty}\left(\lambda^{j}\right)^{2+\eta} P_{j}\left(\frac{x}{\lambda^{j}}\right) .
$$

Since $Q_{k}(x)$ are of the form $r^{3 / 2} B^{*}(\theta)+r^{2} \widetilde{B}^{*}(\theta)$, the limit function is also of the same form, and we denote it by

$$
Q(x) \equiv r^{3 / 2} B(\theta)+r^{2} \widetilde{B}(\theta) .
$$


We then have

$$
\left|Q(x)-Q_{k}(x)\right| \leqslant \sum_{j=k+1}^{\infty}\left(\lambda^{j}\right)^{2+\eta}\left[\left|\frac{x}{\lambda^{j}}\right|^{3 / 2}+\left|\frac{x}{\lambda^{j}}\right|^{2}\right] \leqslant C\left\{\left(\lambda^{k}\right)^{1 / 2+\eta}|x|^{3 / 2}+\left(\lambda^{k}\right)^{\eta}|x|^{2}\right\} .
$$

Therefore,

$$
\left|\varphi_{0}(x)-Q(x)\right| \leqslant C\left(\lambda^{k}\right)^{2+\eta} \quad \text { for }|x|<\lambda^{k} .
$$

For each $|x|<\lambda$, we choose $k$ such that $\lambda^{k+1}<|x| \leqslant \lambda^{k}$. Then the above inequality implies that

$$
\left|\varphi_{0}(x)-Q(x)\right| \leqslant C|x|^{2+\eta}
$$

and the proof of Lemma 5.2 is complete.

Remark 5.1. The above proof also shows that

$$
\left|\nabla\left(\varphi_{0}(x)-Q(x)\right)\right| \leqslant C|x|^{1+\eta} .
$$

Remark 5.2. The iterative argument used in the proof of Lemma 5.2 is similar to the iterative argument used in the proof of Lemma 2.4 of [12].

Lemma 5.3 Suppose $G_{j}$ is the solution of the following system:

$$
\begin{aligned}
& G_{j} \in H^{2}\left(B_{1}(0) \backslash \Gamma_{j}\right), \\
& \Delta^{2} G_{j}=0 \quad \text { in } B_{1}(0) \backslash \Gamma_{j}, \\
& G_{j}=\frac{\partial G_{j}}{\partial n}=0 \quad \text { on both sides of } \Gamma_{j}, \\
& G_{j}=\varphi_{j}, \quad \frac{\partial G_{j}}{\partial n}=\frac{\partial \varphi_{j}}{\partial n} \quad \text { on rest of } \partial\left(B_{1}(0) \backslash \Gamma_{j}\right),
\end{aligned}
$$

where $\Gamma_{j}: x_{2}=\frac{1}{2} f^{\prime \prime}(0) \lambda^{j} \varepsilon x_{1}^{2},-2<x_{1}<0$, and

$$
\left|f^{\prime \prime}(0)\right| \leqslant 1, \quad\left\|\varphi_{j}\right\|_{C^{1}\left(\bar{B}_{1}\right)} \leqslant 1 .
$$

Then, for any small $\delta>0$ there exist sufficiently small $\varepsilon$ and $\lambda$ such that the corresponding $G_{j}$ satisfies:

$$
\begin{gathered}
\left|G_{j}(x)-P_{j}(x)\right| \leqslant \frac{1}{2} \lambda^{3-\delta} \quad \text { for }|x|<2 \lambda, \\
\left|\nabla G_{j}(x)-\nabla P_{j}(x)\right| \leqslant \frac{1}{2} \lambda^{2-\delta} \quad \text { for }|x|<2 \lambda,
\end{gathered}
$$

where

$$
\begin{aligned}
& \left.P_{j}(x)=r^{3 / 2}\left(A_{1}^{j} B_{1}(\theta)+A_{2}^{j} B_{2}(\theta)+A_{2}^{j} \varepsilon \mu_{j} r \dot{B}_{2}(\theta)\right)\right)+r^{2} A_{3}^{j} B_{3}(\theta) \\
& +r^{5 / 2}\left(A_{4}^{j} B_{4}(\theta)+A_{5}^{j} B_{5}(\theta)\right)-r^{5 / 2} \frac{3}{2} A_{2}^{j} \varepsilon \mu_{j} B_{1}(\theta)
\end{aligned}
$$

for all $j \geqslant 0,0 \leqslant \mu_{j}<1$. 
Proof. Under the above assumptions it is clear that

$$
\left|G_{j}\right| \leqslant C|x|^{3 / 2}, \quad\left|\nabla G_{j}\right| \leqslant C|x|^{1 / 2}, \quad\left[\nabla G_{j}\right]_{C^{1 / 2}\left(B_{1 / 2}\right)} \leqslant C .
$$

Let $\widetilde{G}_{j}$ be the solution of the following problem

$$
\begin{aligned}
& \widetilde{G}_{j} \in H^{2}\left(B_{1 / 2}(0) \backslash\{\theta= \pm \pi\}\right), \\
& \Delta^{2} \widetilde{G}_{j}=0 \quad \text { in } B_{1 / 2}(0) \backslash\{\theta= \pm \pi\}, \\
& \widetilde{G}_{j}=\frac{\partial \widetilde{G}_{j}}{\partial n}=0 \quad \text { on }\{\theta= \pm \pi\}, \\
& \widetilde{G}_{j}=G_{j}, \quad \frac{\partial \widetilde{G}_{j}}{\partial n}=\frac{\partial G_{j}}{\partial n} \quad \text { on rest of } \partial\left(B_{1 / 2}(0) \backslash\{\theta= \pm \pi\}\right) .
\end{aligned}
$$

By scaling and using $C^{2+\alpha}$ estimates as before, we get

$$
|x|^{-3 / 2} \varepsilon^{-1}\left|G_{j}-\widetilde{G}_{j}\right|+|x|^{-1 / 2}\left|\nabla\left(G_{j}-\widetilde{G}_{j}\right)\right| \leqslant C \varepsilon \quad \text { if }|\theta \pm \pi| \leqslant 2 \varepsilon .
$$

Then by maximum principle (Theorem 2.2),

$$
\left\|G_{j}-\widetilde{G}_{j}\right\|_{L^{p}\left(B_{1 / 2}\right)} \leqslant C \varepsilon .
$$

For any $\beta<1$, we use $C^{3 / 2}$ regularity and interpolation, and take $p$ to be large enough to obtain

$$
\left|G_{j}-\widetilde{G}_{j}\right| \leqslant C \varepsilon^{\beta}, \quad\left|\nabla\left(G_{j}-\widetilde{G}_{j}\right)\right| \leqslant C \varepsilon^{\beta / 3}
$$

By (1.11), we have

$$
\left|\widetilde{G}_{j}-P(x)\right| \leqslant C r^{3}, \quad\left|\nabla\left(\widetilde{G}_{j}-P(x)\right)\right| \leqslant C r^{2},
$$

where

$$
P(x)=r^{3 / 2} B(\theta)+r^{2} \widetilde{B}(\theta)+r^{5 / 2} \widetilde{\widetilde{B}}(\theta) .
$$

Notice that the extra terms in $P_{j}(x)$ are of order $\varepsilon$. Choosing $\varepsilon$ and $\lambda$ such that

$$
C \varepsilon^{\beta}+C(2 \lambda)^{3}<\frac{1}{4} \lambda^{3-\delta}, \quad C \varepsilon^{\beta / 3}+C(2 \lambda)^{2}<\frac{1}{4} \lambda^{2-\delta},
$$

the proof is now complete.

Proof of Theorem 5.1. We shall modify the proof of Lemma 5.2 for $C^{2}$ expansion to obtain $C^{5 / 2}$ expansion. The $P_{j}$ will be of the form

$$
\begin{aligned}
P_{j}(x)= & \left.\left\{r^{3 / 2}\left(A_{1}^{j} B_{1}(\theta)+A_{2}^{j} B_{2}(\theta)+A_{2}^{j} \varepsilon \mu_{j} r \dot{B}_{2}(\theta)\right)\right)\right\} \\
& +\left\{r^{2} A_{3}^{j} B_{3}(\theta)+r^{5 / 2}\left(A_{4}^{j} B_{4}(\theta)+A_{5}^{j} B_{5}(\theta)\right)-r^{5 / 2} \frac{3}{2} A_{2}^{j} \varepsilon \mu_{j} B_{1}(\theta)\right\}, \\
\equiv & P_{j}^{1}(x)+P_{j}^{2}(x)
\end{aligned}
$$

obtained from Lemma 5.3. 
Notice that the terms $r^{5 / 2} \cos \frac{3}{2} \theta$ in $P_{j}^{1}$ and $P_{j}^{2}$ cancel out, and the term $r^{5 / 2} \cos \frac{1}{2} \theta$ (in $P_{j}^{1}$ and $\left.P_{j}^{2}\right)$ is biharmonic. Thus $P_{j}$ still satisfies the biharmonic equation: $\Delta^{2} P_{j} \equiv 0$.

We define $\varphi_{k}$ inductively as

$$
\begin{aligned}
\varphi_{k+1} & =\frac{1}{\lambda^{3-\delta}}\left(\varphi_{k}(\lambda x)-P_{k}(\lambda x)\right) \\
& =\left(\lambda^{-(3-\delta)}\right)^{k+1} \varphi_{0}\left(\lambda^{k+1} x\right)-\sum_{j=0}^{k}\left(\lambda^{-(3-\delta)}\right)^{j+1} P_{k-j}\left(\lambda^{j+1} x\right),
\end{aligned}
$$

where $\mu_{k-j}$ are still to be determined.

Instead of two lines $\theta= \pm\left(\pi-2 \varepsilon \lambda^{k}\right)$, we now use the two $C^{\infty}$ curves

$$
\Gamma_{ \pm}^{k}: \quad x_{2}=\frac{1}{2} \varepsilon \lambda^{k} f^{\prime \prime}(0) x_{1}^{2} \pm \varepsilon^{1+\alpha} \lambda^{(1+\alpha) k} x_{1}^{2}, \quad-2<x_{1}<0
$$

which are $\varepsilon^{1+\alpha} \lambda^{(1+\alpha) k} x_{1}^{2}$ close to the original curve (instead of just $\varepsilon \lambda^{k} x_{1}$ close to the original curve). Then, using (5.4), we find that on $\Gamma_{ \pm}^{k+1}$, as well as on any arc $|x|=$ const. in the thin region connecting $\Gamma_{+}^{k+1}$ and $\Gamma_{-}^{k+1}$,

$$
\begin{aligned}
& \left|\left(\lambda^{-(3-\delta)}\right)^{k+1} \varphi_{0}\left(\lambda^{k+1} x\right)\right| \leqslant C \varepsilon^{2(1+\alpha)} \lambda^{(k+1)(1 / 2+2 \alpha+\delta)}|x|^{3 / 2}, \\
& \left|\nabla\left(\left(\lambda^{-(3-\delta)}\right)^{k+1} \varphi_{0}\left(\lambda^{k+1} x\right)\right)\right| \leqslant C \varepsilon^{1+\alpha} \lambda^{(k+1)(\alpha+\delta-1 / 2)}|x|^{1 / 2}, \\
& {\left[\nabla\left(\left(\lambda^{-(3-\delta)}\right)^{k+1} \varphi_{0}\left(\lambda^{k+1} x\right)\right)\right]_{C \sigma\left(\Gamma_{ \pm}^{k+1} \cap\{|x|<R\}\right)} \leqslant C \varepsilon^{(1+\alpha)(1-\sigma)} R^{1 / 2-\sigma}}
\end{aligned}
$$

provided $0<\delta<1 / 2, \alpha+\delta>1 / 2,0<\sigma \leqslant \sigma_{0}$, where

$$
\sigma_{0}=\frac{\alpha+\delta-1 / 2}{2+\alpha}
$$

cf. the proof of (5.18)-(5.20).

Next, we estimate the sum

$$
\sum\left(\lambda^{-(3-\delta)}\right)^{j+1} P_{k-j}\left(\lambda^{j+1} x\right)=\sum\left(\lambda^{-(3-\delta)}\right)^{j+1} P_{k-j}^{1}\left(\lambda^{j+1} x\right)+\sum\left(\lambda^{-(3-\delta)}\right)^{j+1} P_{k-j}^{2}\left(\lambda^{j+1} x\right)
$$

on the curves $\Gamma_{ \pm}^{k+1}$ and in the thin region lying between them. The second derivatives of those terms involving $r^{2}$ and $r^{5 / 2}$ are bounded. Therefore, we can follow the calculations as in the case for $C^{2}$ expansion to conclude that on $\Gamma_{ \pm}^{k+1}$ as well as any arc $|x|=$ const. which lies in the thin region lying between $\Gamma_{+}^{k+1}$ and $\Gamma_{-}^{k+1}$,

$$
\begin{aligned}
& \left|\sum\left(\lambda^{-(3-\delta)}\right)^{j+1} P_{k-j}^{2}\left(\lambda^{j+1} x\right)\right| \leqslant C \varepsilon^{2} \lambda^{k(1+\delta)}|x|^{3 / 2} \\
& \left|\nabla\left(\sum\left(\lambda^{-(3-\delta)}\right)^{j+1} P_{k-j}^{2}\left(\lambda^{j+1} x\right)\right)\right| \leqslant C \varepsilon \lambda^{k \delta}|x|^{1 / 2}
\end{aligned}
$$

furthermore,

$$
\begin{gathered}
{\left[\nabla\left(\sum\left(\lambda^{-(3-\delta)}\right)^{j+1} P_{k-j}^{2}\left(\lambda^{j+1} x\right)\right)\right]_{C^{\sigma}\left(\Gamma_{ \pm}^{k+1} \cap\{|x|<R\}\right)} \leqslant C \varepsilon^{(1-\sigma)} \lambda^{k(\delta-2 \sigma)} R^{1 / 2-\sigma}} \\
\leqslant C \varepsilon^{(1-\sigma)} R^{1 / 2-\sigma}
\end{gathered}
$$


provided $\sigma \leqslant \delta / 2$

Next, we split $P_{k}^{1}$ into two parts.

$$
\begin{aligned}
P_{k}^{1}(x) & =r^{3 / 2} A_{1}^{k} B_{1}(\theta)+r^{3 / 2} A_{2}^{k}\left(B_{2}(\theta)+\varepsilon \mu_{k} r \dot{B}_{2}(\theta)\right) \\
& \equiv P_{k}^{11}(x)+P_{k}^{12}(x) .
\end{aligned}
$$

Since

$$
\left.B_{1}(\theta)\right|_{\theta= \pm \pi}=\left.\frac{\partial}{\partial \theta} B_{1}(\theta)\right|_{\theta= \pm \pi}=\left.\frac{\partial^{2}}{\partial \theta^{2}} B_{1}(\theta)\right|_{\theta= \pm \pi}=0
$$

we have

$$
B_{1}(\theta)=O\left(|\theta \pm \pi|^{3}\right), \quad \frac{\partial}{\partial \theta} B_{1}(\theta)=O\left(|\theta \pm \pi|^{2}\right)
$$

Using the computation for $C^{2}$ expansion, we find that the estimates (5.42)-(5.44) are valid for $\sum\left(\lambda^{-(3-\delta)}\right)^{j+1} P_{k-j}^{11}\left(\lambda^{j+1} x\right)$.

To estimate $P_{k}^{12}$, notice that $-x_{1}=r+O\left(r^{2}\right)$ on the curve $x_{2}=\frac{1}{2} f^{\prime \prime}(0) x_{1}^{2}, x_{1}<0$. This curve can be rewritten in polar coordinates:

$$
\theta= \pm \pi-\frac{1}{2} f^{\prime \prime}(0) r+O\left(r^{2}\right)
$$

We take $\mu_{j}=\lambda^{j} f^{\prime \prime}(0) / 2$ in order to make the curves $\Gamma_{ \pm}^{k} \varepsilon^{1+\alpha} \lambda^{(1+\alpha) k} r^{2}$ close to the curves $\theta= \pm \pi-\varepsilon \mu_{k} r$. Since $\dot{B}_{2}(\theta)=(\partial / \partial \theta) B_{2}(\theta)=\frac{3}{2} \cos \frac{3}{2} \theta+\frac{1}{2} \cos \frac{1}{2} \theta$, we have

$$
\left.B_{2}(\theta)\right|_{\theta= \pm \pi}=\left.\frac{\partial}{\partial \theta} B_{2}(\theta)\right|_{\theta= \pm \pi}=\left.\frac{\partial^{3}}{\partial \theta^{3}} B_{2}(\theta)\right|_{\theta= \pm \pi}=0,\left.\quad \frac{\partial^{2}}{\partial \theta^{2}} B_{2}(\theta)\right|_{\theta= \pm \pi}= \pm 2
$$

and

$$
\begin{aligned}
& B_{2}(\theta)+\left.\varepsilon \mu_{k} r \dot{B}_{2}(\theta)\right|_{\theta= \pm \pi}=0, \\
& \left.\frac{\partial}{\partial \theta}\left(B_{2}(\theta)+\varepsilon \mu_{k} r \dot{B}_{2}(\theta)\right)\right|_{\theta= \pm \pi}= \pm 2 \varepsilon \mu_{k} r \\
& \left.\frac{\partial^{2}}{\partial \theta^{2}}\left(B_{2}(\theta)+\varepsilon \mu_{k} r \dot{B}_{2}(\theta)\right)\right|_{\theta= \pm \pi}= \pm 2, \\
& \left|\frac{\partial^{3}}{\partial \theta^{3}}\left(B_{2}(\theta)+\varepsilon \mu_{k} r \dot{B}_{2}(\theta)\right)\right|_{|\theta \pm \pi|<2 \varepsilon \mu_{k}} \leqslant C \varepsilon \mu_{k} .
\end{aligned}
$$

Therefore

$$
\begin{aligned}
B_{2}(\theta)+\left.\varepsilon \mu_{k} r \dot{B}_{2}(\theta)\right|_{\theta= \pm \pi-\varepsilon \mu_{k} r} & = \pm\left(2 \varepsilon \mu_{k} r\right)\left(-\varepsilon \mu_{k} r\right)+\frac{1}{2} \cdot( \pm 2) \cdot\left(-\varepsilon \mu_{k} r\right)^{2}+O\left(\left|\varepsilon \mu_{k} r\right|^{3}\right) \\
& =O\left(\left|\varepsilon \mu_{k} r\right|^{2}\right), \\
\left.\frac{\partial}{\partial \theta}\left[B_{2}(\theta)+\varepsilon \mu_{k} r \dot{B}_{2}(\theta)\right]\right|_{\theta= \pm \pi-\varepsilon \mu_{k} r} & = \pm 2 \varepsilon \mu_{k} r \pm 2\left(-\varepsilon \mu_{k} r\right)+O\left(\left|\varepsilon \mu_{k} r\right|^{2}\right)=O\left(\left|\varepsilon \mu_{k} r\right|^{2}\right), \\
\left.\frac{\partial}{\partial r}\left[B_{2}(\theta)+\varepsilon \mu_{k} r \dot{B}_{2}(\theta)\right]\right|_{\theta= \pm \pi-\varepsilon \mu_{k} r} & =\left.\varepsilon \mu_{k} \dot{B}_{2}(\theta)\right|_{\theta= \pm \pi-\varepsilon \mu_{k} r}=\varepsilon \mu_{k} O\left(\left|\varepsilon \mu_{k} r\right|\right) .
\end{aligned}
$$


Rewriting these estimates in terms of $P_{k}^{12}$, we have

$$
\begin{aligned}
& \left.\left|P_{k}^{12}(x)\right|\right|_{\theta= \pm \pi-\varepsilon \mu_{k} r} \leqslant C \varepsilon^{2} \mu_{k}^{2} r^{7 / 2}, \\
& \left.\left|\nabla P_{k}^{12}(x)\right|\right|_{\theta= \pm \pi-\varepsilon \mu_{k} r} \leqslant C \varepsilon^{2} \mu_{k}^{2} r^{5 / 2} .
\end{aligned}
$$

From the definition of $P_{k}^{12}$ it is clear that

$$
\left|D^{2} P_{k}^{12}(x)\right| \leqslant C|x|^{-1 / 2} \quad \text { for }|x|<2 .
$$

Recall that $\Gamma_{ \pm}^{k}$ is $\varepsilon^{1+\alpha} \lambda^{(1+\alpha) k} r^{2}$ close to the curve $\theta= \pm \pi-\varepsilon \mu_{k} r$. Using (5.45)-(5.47), we can then derive the estimates (similarly to $(5.39)-(5.41)$ )

$$
\begin{aligned}
& \left|\sum\left(\lambda^{-(3-\delta)}\right)^{j+1} P_{k-j}^{12}\left(\lambda^{j+1} x\right)\right| \leqslant C \varepsilon^{2} \lambda^{(k+1)(1 / 2+\delta)}|x|^{3 / 2}, \\
& \left|\nabla\left(\sum\left(\lambda^{-(3-\delta)}\right)^{j+1} P_{k-j}^{12}\left(\lambda^{j+1} x\right)\right)\right| \leqslant C \varepsilon^{1+\alpha} \lambda^{(k+1)(\alpha+\delta-1 / 2)}|x|^{1 / 2}, \\
& {\left[\nabla\left(\sum\left(\lambda^{-(3-\delta)}\right)^{j+1} P_{k-j}^{12}\left(\lambda^{j+1} x\right)\right)\right]_{C^{\sigma}\left(\Gamma_{ \pm}^{k+1} \cap\{|x|<R\}\right)} \leqslant C \varepsilon^{(1+\alpha)(1-\sigma)} R^{1 / 2-\sigma},}
\end{aligned}
$$

in the thin region bounded by $\Gamma_{ \pm}^{k+1}$.

Combining all these estimates, we find that

$$
\left.\left|\varphi_{k+1}(x)\right|\right|_{\Gamma_{ \pm}^{k+1}} \leqslant C \varepsilon^{2},\left.\quad\left|\nabla \varphi_{k+1}(x)\right|\right|_{\Gamma_{ \pm}^{k+1}} \leqslant C \varepsilon, \quad\left[\nabla \varphi_{k+1}\right]_{C^{\sigma}\left(\Gamma_{ \pm}^{k+1}\right)} \leqslant C \varepsilon^{1-\sigma}
$$

By Theorem 2.2, we then have

$$
\left\|\varphi_{k+1}-G_{k+1}\right\|_{L^{p}\left(B_{1}\right)} \leqslant C \varepsilon
$$

where $G_{k+1}$ is given in Lemma 5.3. From this, we can argue in the same way as before (using Lemma 5.4 below in deriving Hölder estimates for $\left.\nabla\left(\varphi_{k+1}-G_{k+1}\right)\right)$ to conclude that, for any $\beta<1$,

$$
\begin{aligned}
& \left|\varphi_{k+1}-G_{k+1}\right| \leqslant C \varepsilon^{\beta} \\
& \left|\nabla\left(\varphi_{k+1}-G_{k+1}\right)\right| \leqslant C \varepsilon^{\beta \sigma /(1+\sigma)} .
\end{aligned}
$$

Combining this with Lemma 5.3, we conclude that

$$
\begin{aligned}
& \left|\varphi_{k+1}-P_{k+1}\right| \leqslant \lambda^{3-\delta}, \\
& \left|\nabla\left(\varphi_{k+1}-P_{k+1}\right)\right| \leqslant \lambda^{2-\delta} .
\end{aligned}
$$

We now proceed as in the proof of (5.28)-(5.30) (with $\lambda^{2+\eta}$ replaced by $\lambda^{3-\delta}$ ) to establish the estimate

$$
\begin{aligned}
\varphi_{0}(x)=r^{3 / 2} & \left(A_{1} B_{1}(\theta)+A_{2} B_{2}(\theta)\right)+r^{2} A_{3} B_{3}(\theta) \\
& +r^{5 / 2}\left[A_{4} B_{4}(\theta)+A_{5} B_{5}(\theta)-2 A_{2} \varepsilon f^{\prime \prime}(0) \cos \frac{1}{2} \theta\right]+O\left(r^{3-\delta}\right),
\end{aligned}
$$


which, when written in terms of the original variables, becomes

$$
\begin{aligned}
\varphi(x)=r^{3 / 2} & \left(A_{1} B_{1}(\theta)+A_{2} B_{2}(\theta)\right)+r^{2} A_{3} B_{3}(\theta) \\
& +r^{5 / 2}\left[A_{4} B_{4}(\theta)+A_{5} B_{5}(\theta)-2 A_{2} f^{\prime \prime}(0) \cos \frac{1}{2} \theta\right]+O\left(r^{3-\delta}\right),
\end{aligned}
$$

with different coefficients $A_{3}, A_{4}, A_{5}$. This completes the proof of (1.14) (with $\eta=\delta$ ).

Remark 5.3. Theorem 5.1 is new even if $\Gamma$ is a parabola $x_{2}=x_{1}^{2}$.

Remark 5.4. If $f^{\prime}(0) \neq 0$ then, in Theorem 5.1, the second derivative $f^{\prime \prime}(0)$ should be replaced by the curvature $\kappa$ at 0 and $B_{i}(\theta)$ should be replaced by $B_{i}\left(\theta-\theta_{0}\right)$, where $\theta_{0}=f^{\prime}(0)$.

Remark 5.5. As in the case of Remark 5.1, the proof of Theorem 5.1 shows that (1.14) can be differentiated, with

$$
\nabla\left(O\left(r^{3-\eta}\right)\right)=O\left(r^{2-\eta}\right)
$$

The same remark applies to (1.13), with $\nabla\left(O\left(r^{2-\eta}\right)\right)=O\left(r^{1-\eta}\right)$ and the proof can be given by the method of $\S 3$ using (2.20).

The next lemma establishes (as a special case) the estimate (5.21) which was needed in the proof of Lemma 5.2.

Lemma 5.4 Let $0<\alpha<1$, and let $f_{j}=f_{j}^{\tau}\left(x_{1}\right)(j=1,2)$ be curves satisfying:

$$
\begin{gathered}
f_{j}(0)=f_{j}^{\prime}(0)=0, \quad\left[f_{j}^{\prime}\right]_{C^{\alpha}[-2,0]} \leqslant 1, \\
\tau x_{1} \leqslant f_{1}\left(x_{1}\right) \leqslant f_{2}\left(x_{2}\right) \leqslant-\tau x_{1} \quad \text { for }-2<x_{1}<0,
\end{gathered}
$$

where $0<\tau<1$. Let $\varphi \in H^{2}\left(B_{2} \backslash\left\{f_{1}\left(x_{1}\right) \leqslant x_{2} \leqslant f_{2}\left(x_{2}\right), x_{1}<0\right\}\right)$ satisfy

$$
\begin{aligned}
& \Delta^{2} \varphi=0 \quad \text { in } B_{2} \backslash\left\{f_{1}\left(x_{1}\right) \leqslant x_{2} \leqslant f_{2}\left(x_{2}\right), x_{1}<0\right\}, \\
& |\nabla \varphi| \leqslant 1 \quad \text { in } B_{2}, \\
& \varphi(0)=0, \quad \nabla \varphi(0)=0, \\
& {[\nabla \varphi]_{C^{\sigma}\left(\left\{\left|x_{2}\right| \leqslant-\tau x_{1}\right\} \cap\left\{|x|<, x_{1}<0\right\}\right) \leqslant 1 .}}
\end{aligned}
$$

Then, for any $\sigma<1 / 2$,

$$
|\varphi(x)| \leqslant C|x|^{1+\sigma}
$$

where the constant $C$ depends only on $\sigma$ and $\alpha$, but not on the $f_{j}$ and $\tau$.

Proof. From (5.52) and (5.53) we deduce that

$$
|\varphi(x)| \leqslant|x| \text { in } B_{2} .
$$

Next, (5.53) and (5.54) imply that

$$
|\nabla \varphi(x)| \leqslant|x|^{\sigma} \quad \text { for }\left|x_{2}\right| \leqslant-\tau x_{1}, \quad x_{1}<0 \quad \text { and }|x|<2 \text {, }
$$


and therefore

$$
|\varphi(x)| \leqslant|x|^{1+\sigma} \quad \text { for }\left|x_{2}\right| \leqslant-\tau x_{1}, \quad x_{1}<0 \quad \text { and }|x|<2 \text {. }
$$

Consider the function

$$
Z_{\delta}(x)=\frac{\varphi(x)}{|x|^{1+\sigma}+\delta|x|} \quad \forall \delta>0 .
$$

By (5.56), sup $\left|Z_{\delta}(x)\right|<+\infty$ for any $\delta>0$. We claim that $|x|<1$

$$
\sup _{|x|<1}\left|Z_{\delta}(x)\right| \leqslant C
$$

for some constant $C$ independent of $\delta, \tau$ and $f_{j}$; once this is proved, we can then finish the proof of the lemma by letting $\delta \rightarrow 0$.

Suppose (5.60) is not true. Then there exist sequences $\varphi=\varphi_{n}, f_{j}=f_{n j}(j=1,2)$, $\delta_{n} \rightarrow 0, x_{n} \rightarrow 0, \tau_{n}\left(\tau_{n}\right.$ may go to 0$)$ such that

$$
C_{n}=\sup _{|x|<1}\left|Z_{\delta_{n}}(x)\right|=\left|Z_{\delta_{n}}\left(x_{n}\right)\right| \rightarrow \infty .
$$

Define $G_{n}(\xi)$ by:

$$
\varphi_{n}(x)=C_{n}\left(R_{n}^{1+\sigma}+\delta_{n} R_{n}\right) G_{n}(\xi) \quad \text { where } \quad x=R_{n} \xi, \quad R_{n}=\left|x_{n}\right| .
$$

Then

$$
\left|G_{n}(\xi)\right| \leqslant \frac{\left|\varphi_{n}(x)\right|}{C_{n}\left(R_{n}^{1+\sigma}+\delta_{n} R_{n}\right)} \leqslant \frac{|\xi|^{1+\sigma} R_{n}^{1+\sigma}+\delta_{n}|\xi| R_{n}}{\left(R_{n}^{1+\sigma}+\delta_{n} R_{n}\right)} \leqslant|\xi|^{1+\sigma}+|\xi|,
$$

and, from (5.58), (5.57) and (5.54) we also have

$$
\begin{aligned}
& \left|G_{n}(\xi)\right| \leqslant \frac{R_{n}^{1+\sigma}}{C_{n}\left(R_{n}^{1+\sigma}+\delta_{n} R_{n}\right)}|\xi|^{1+\sigma} \leqslant \frac{1}{C_{n}}|\xi|^{1+\sigma} \quad \text { for }\left|\xi_{2}\right|<-\tau_{n} \xi_{1},|\xi| \leqslant \frac{2}{R_{n}} \\
& \left|\nabla_{\xi} G_{n}(\xi)\right| \leqslant \frac{R_{n}^{\sigma}}{C_{n}\left(R_{n}^{\sigma}+\delta_{n}\right)}|\xi|^{1 / 2} \leqslant \frac{1}{C_{n}}|\xi|^{1 / 2} \quad \text { for }\left|\xi_{2}\right|<-\tau_{n} \xi_{1},|\xi| \leqslant \frac{2}{R_{n}}
\end{aligned}
$$

and, for any $K>0$,

$$
\left[\nabla_{\xi} G_{n}\right]_{C^{\sigma}\left(\left\{\left|\xi_{2}\right|<-\tau_{n} \xi_{1}\right\} \cap\{|\xi|<K\}\right)} \leqslant \frac{R_{n}^{\sigma}}{C_{n}\left(R_{n}^{\sigma}+\delta_{n}\right)} \leqslant \frac{1}{C_{n}} .
$$

The curve $x_{2}=f_{j}\left(x_{1}\right)(j=1,2)$ under the change of variables $x \rightarrow \xi$ becomes

$$
\xi_{2}=\frac{1}{R_{n}} f_{j}\left(R_{n} \xi_{1}\right), \quad-\frac{2}{R_{n}}<\xi_{1}<0
$$

Under the assumptions of the lemma, we have, for any $K>1$,

$$
\begin{aligned}
& \left\{\frac{1}{R_{n}} f_{1}\left(R_{n} \xi_{1}\right) \leqslant \xi_{2} \leqslant \frac{1}{R_{n}} f_{2}\left(R_{n} \xi_{1}\right)\right\} \cap\{|\xi|<K\} \subset\left\{\left|\xi_{2}\right| \leqslant-R_{n}^{\alpha} K^{\alpha} \xi_{1}\right\}, \\
& \left\{\frac{1}{R_{n}} f_{1}\left(R_{n} \xi_{1}\right) \leqslant \xi_{2} \leqslant \frac{1}{R_{n}} f_{2}\left(R_{n} \xi_{1}\right)\right\} \subset\left\{\left|\xi_{2}\right| \leqslant-\tau_{n} \xi_{1}\right\} .
\end{aligned}
$$


Therefore, for $\beta_{n}=\min \left(R_{n}^{\alpha} K^{\alpha}, \tau_{n}\right)$, we have

$$
\left\{\frac{1}{R_{n}} f_{1}\left(R_{n} \xi_{1}\right) \leqslant \xi_{2} \leqslant \frac{1}{R_{n}} f_{2}\left(R_{n} \xi_{1}\right)\right\} \cap\{|\xi|<K\} \subset\left\{\left|\xi_{2}\right| \leqslant-\beta_{n} \xi_{1}\right\}
$$

Just as in the proof of Lemma 3.2, we can now apply Theorem 9.3 (ii) with boundary given by the rays $\left|\xi_{2}\right|=-\beta_{n} \xi_{1}(|\xi|<K)$ to conclude that (for a subsequence and any $K>1$ ) $G_{n}(\xi) \rightarrow G(\xi)$, where $G(\xi)$ satisfies

$$
\begin{aligned}
& \Delta^{2} G=0 \quad \text { in } \mathbb{R}^{2} \backslash \bar{S}_{0}, \\
& G=\frac{\partial G}{\partial \nu}=0 \quad \text { from both sides of } S_{0}, \\
& |G(\xi)| \leqslant|\xi|^{1+\sigma}+|\xi| \quad \text { in } \mathbb{R}^{2}, \\
& |G(e)|=1 \quad \text { where } e=\lim \frac{x_{n}}{R_{n}},
\end{aligned}
$$

and where $S_{0}=\left\{\left(\xi_{1}, 0\right) ; \xi_{1}<0\right\}$. This is a contradiction to the Liouville theorem stated in the following lemma.

Lemma 5.5 If $G$ satisfies (5.61)-(5.63), then $G(\xi) \equiv 0$.

Proof. The proof is similar to that of Lemma 3.3. Let $\widetilde{G} \in H^{2}\left(B_{1} \backslash S_{0}\right)$ be a solution of

$$
\begin{aligned}
& \Delta^{2} \widetilde{G}=0 \quad \text { in } B_{1} \backslash S_{0}, \\
& \widetilde{G}=\frac{\partial \widetilde{G}}{\partial \nu}=0 \quad \text { from both sides of } S_{0}, \\
& \widetilde{G}=G, \quad \frac{\partial \widetilde{G}}{\partial \nu}=\frac{\partial G}{\partial \nu} \quad \text { on } \partial B_{1} .
\end{aligned}
$$

Then $|\widetilde{G}-G| \leqslant C|\xi|$ and $|\nabla(\widetilde{G}-G)| \leqslant C$ near $\xi=0$. Applying the maximum principle (Theorem 2.3) to $\widetilde{G}-G$ in the domain $B_{1} \backslash\left(S_{0} \cup B_{\varepsilon}\right)$ and then letting $\varepsilon \rightarrow 0$ we conclude that $\widetilde{G}-G \equiv 0$. Since $\widetilde{G}$ has an expansion at the origin, this expansion is valid also for $G(\xi):$

$$
G(\xi)=\sum_{k=1}^{\infty} r^{k / 2+1} B_{k}(\theta) \quad(r=|\xi|)
$$

near $\xi=0$. Introduce the function

$$
H(\xi)=G(\xi)-r^{3 / 2} B_{1}(\theta)-r^{2} B_{2}(\theta)
$$

Then

$$
|H(\xi)| \leqslant C|\xi|^{5 / 2} \quad \text { near } \xi=0, \quad\left|G(\xi)-r^{3 / 2} B_{1}(\theta)\right| \leqslant C|\xi|^{3 / 2} \quad \text { near } \xi=\infty
$$

Since $r^{2} B_{2}(\theta)=c \xi_{2}^{2}, \partial / \partial \xi_{1}\left[r^{2} B_{2}(\theta)\right]=0$. The function

$$
\Phi(\xi)=\frac{\partial H}{\partial \xi_{1}}=\frac{\partial G}{\partial \xi_{1}}-\frac{\partial}{\partial \xi_{1}}\left[r^{3 / 2} B_{1}(\theta)\right]
$$


is biharmonic in $\mathbb{R}^{2} \backslash S_{0}$ and satisfies the same zero boundary conditions. It follows that

$$
\left|\nabla^{j} \Phi(\xi)\right| \leqslant C|\xi|^{5 / 2-1-j} \text { near } \xi=0, \quad j=0,1,2,3,
$$

and

$$
\left|\nabla^{j} \Phi(\xi)\right| \leqslant C|\xi|^{3 / 2-1-j} \text { near } \xi=\infty \quad j=0,1,2,3 .
$$

Now we can follow the proof of Lemma 3.3 to conclude that $\Phi \equiv 0$, which immediately implies that $G \equiv 0$.

Remark 5.7. The preceding Liouville theorem does not follow from a general theorem of Kondratév [13; Theorem 11] since one of the assumptions he makes,

$$
\int_{0}^{\infty} \int_{0}^{\infty} r^{\gamma}|G|^{2} r d r d \theta<\infty
$$

is not satisfies for any $\gamma$, in our case.

Remark 5.8. The proof of Lemma 5.2 can be extended to the case where $f \in C^{1+\alpha}$ to yield a different (although more complicated) proof of Theorem 3.1. In (5.16) we need to replace $\lambda^{2+\eta}$ by $\lambda^{1+\eta}$, and $(5.7),(5.8)$ need to be modified by using the fact that $\varphi_{0}$ is $C^{3 / 2}$ and applying (5.2) to $\zeta=\nabla \varphi_{1}$ with $\theta=\sigma<1 / 2$. Finally, (5.9) follows from Lemma 5.4 with $f_{j}\left(x_{1}\right)=\delta_{j}\left|x_{1}\right|^{1+\alpha}, \quad-1<\delta_{2}<\delta_{1}<1$.

\section{The crack propagation model}

In this section we introduce a model of crack propagation. Let $\Omega$ be a domain in $\mathbb{R}^{2}$, representing a homogeneous elastic body. Let $u=\left(u_{i}\right), \varepsilon=\left(\varepsilon_{i j}\right)$ and $s=\left(\sigma_{i j}\right)$ denote the displacement vector, the strain tensor and the stress tensor, respectively. The linear elasticity equations for homogeneous isotropic material consist of the constitutive law

$$
\sigma_{i j}=\frac{E}{1+\nu}\left(\varepsilon_{i j}+\frac{\nu}{1-2 \nu} \varepsilon_{k k} \delta_{i j}\right)
$$

and the equilibrium conditions

$$
\frac{\partial}{\partial x_{j}} \sigma_{i j}=0
$$

provided there are no body forces. Here $E$ is the Young modulus, $\nu$ is the Poisson ratio, and the strain-displacement relations are given by

$$
\varepsilon_{i j}=\frac{1}{2}\left(u_{i, j}+u_{j, i}\right), \quad u_{i, j}=\partial_{j} u_{i} .
$$

Suppose there is initially a crack in $\Omega$, given by a non-intersecting curve $\Gamma_{0}$ with initial point on $\partial \Omega$ and terminal point (the "crack tip") $X_{0}=\left(x_{0}, y_{0}\right)$ inside $\Omega$. Under external forces the crack tip will generally propagate, and we shall denote it by $X(t)$. The crack propagation problem consists of finding the displacement $u$ and path $X(t)$ such that

$$
\sigma_{i j, j}=0 \quad \text { in } \Omega \backslash \Gamma(t)
$$


where

$$
\begin{aligned}
& \Gamma(t)=\Gamma_{0} \cup\{X=X(s), 0 \leqslant s \leqslant t\}, \\
& \sigma_{i j} n_{j}=0 \quad \text { on } \Gamma^{ \pm}(t) \quad\left(\text { no traction on } \Gamma^{ \pm}(t)\right)
\end{aligned}
$$

$\Gamma^{ \pm}(t)$ means both sides of $\Gamma(t),\left(n_{j}\right)$ is the normal to the curve,

$$
u_{i}=\beta_{i} \quad \text { on } \partial_{1} \Omega, \quad \sigma_{i j} n_{j}=g_{i} \quad \text { on } \partial_{2} \Omega
$$

where $\partial \Omega$ is a disjoint union of $\partial_{1} \Omega, \partial_{2} \Omega$, and an appropriate dynamical equation for $X(t)$. Based on [7] [10] [19] [20] [21], Friedman and Liu [9] introduced the following dynamics:

$$
\dot{X}(t)=\frac{v(t)}{\gamma_{0}} \frac{v_{\infty}-v(t)}{v_{\infty}+v(t)} \mathbf{J}(X(t))
$$

where

$$
v(t)=|\dot{X}(t)|, \quad X(0)=X_{0} .
$$

Here $\gamma_{0}, v_{\infty}$ are positive constants and $\mathbf{J}(X(t))$ is described in terms of the $J$-integral

$$
\mathbf{J}_{\gamma}=\int_{\gamma}(W \cdot \vec{n}-\vec{s} \cdot D u) d l
$$

where

$$
W=\frac{1}{2} \sigma_{i j} \varepsilon_{i j}
$$

is the strain energy density and

$$
\vec{s}=\left(s_{i}\right)=\left(\sigma_{i j} n_{j}\right)
$$

is the traction vector; $\gamma$ is a curve in $\Omega \backslash \Gamma(t)$, initiating at $Y$ and terminating at $X, \vec{n}$ is a normal to $\gamma$, and $d l$ is the arc element. It is well known [11] that $\mathbf{J}_{\gamma}$ is independent of the path connecting $Y$ to $X$. Denote by $S_{\varepsilon}(X)$ the circle with center $X$ and radius $\varepsilon$, and set $\Lambda_{\varepsilon}(X(t))=S_{\varepsilon}(X(t)) \cap \Omega$. By the path-independence property of the $J$-integral it follows that

$$
\mathbf{J}(X(t)) \equiv \lim _{\varepsilon \rightarrow 0} \int_{\Lambda_{\varepsilon}(X(t))}(W \cdot \vec{n}-\vec{s} \cdot D u) d l
$$

is well defined, and this is the function we use in (6.8); here $\vec{n}$ is the outward normal to the circles $S_{\varepsilon}(X(t))$. Taking the absolute value in (6.8) we get

$$
1=\frac{1}{\gamma_{0}} \frac{v_{\infty}-|\dot{X}(t)|}{v_{\infty}+|\dot{X}(t)|}|\mathbf{J}(X(t))|,
$$

or

$$
\mid \dot{X}(t)) \mid=v_{\infty} \frac{\left(|\mathbf{J}(X(t))|-\gamma_{0}\right)_{+}}{|\mathbf{J}(X(t))|+\gamma_{0}} .
$$

Hence (6.8) can also be written in the form

$$
\dot{X}(t)=h(|\mathbf{J}(X(t))|) \mathbf{J}(X(t))
$$


where

$$
h(s)=\frac{v_{\infty}}{s} \frac{\left(s-\gamma_{0}\right)_{+}}{s+\gamma_{0}} .
$$

Note that the crack cannot propagate unless $|J(X(t))|$ is larger than $\gamma_{0}$. In particular, if $|J(X(0))| \leqslant \gamma_{0}$, then the crack does not propagate, and $\Gamma(t) \equiv \Gamma_{0}$. Hence in the sequel we shall always assume that $|J(X(0))|>\gamma_{0}$.

As in [9] we can express the stress components in terms of the stress function $\varphi$ (which is determined up to an additive linear function):

$$
\sigma_{11}=\frac{\partial^{2} \varphi}{\partial y^{2}}=\varphi_{22}, \sigma_{12}=-\frac{\partial^{2} \varphi}{\partial x \partial y}=-\varphi_{12}, \sigma_{22}=\frac{\partial^{2} \varphi}{\partial x^{2}}=\varphi_{11}
$$

Then the system $(6.1)-(6.6)$ becomes:

$$
\begin{aligned}
& \Delta^{2} \varphi=0 \quad \text { in } \Omega \backslash \bar{\Gamma}(t), \\
& \varphi=0, \quad \frac{\partial \varphi}{\partial n}=0 \quad \text { on } \Gamma(t), \text { from both sides, } \\
& \Gamma(t)=\Gamma_{0} \cup\{X(s), 0 \leqslant s \leqslant t\} .
\end{aligned}
$$

For definiteness we take boundary conditions (cf. [9])

$$
\varphi=g, \quad \frac{\partial \varphi}{\partial n}=h \quad \text { on } \partial \Omega
$$

We finally recall that

$$
\mathbf{J}(X(t))=\left(J_{1}(X(t)), J_{2}(X(t))\right)
$$

can be computed in the form

$$
J_{i}(X(t))=\frac{1-\nu^{2}}{2 E} \lim _{\varepsilon \rightarrow 0} \int_{\Lambda_{\varepsilon}(X(t))}\left[(\Delta \varphi)^{2} n_{i}-2 \vec{s} \cdot \vec{\Phi}_{i}\right] d l,
$$

where

$$
\begin{aligned}
& s_{1}=\varphi_{22} n_{1}-\varphi_{12} n_{2}, \quad s_{2}=-\varphi_{12} n_{1}+\varphi_{11} n_{2}, \\
& \vec{\Phi}_{1}=\left(\Delta \varphi,(\Delta \varphi)^{c}\right), \vec{\Phi}_{2}=\left(-(\Delta \varphi)^{c}, \Delta \varphi\right)
\end{aligned}
$$

and $(\Delta \varphi)^{c}$ is the harmonic conjugate of $\Delta \varphi$ determined up to an additive constant (the constant disappears in the limit in (6.17).

Definition. The crack problem, Problem (C), is the problem of solving the system $(6.13)-(6.18)$.

\section{Reformulation of the crack propagation problem}

In this section we reformulate the crack problem by first replacing the dynamic formulation (6.11) by a geometric condition, and then replacing the latter by the condition (1.17). 
We assume that $(\varphi, \Gamma)$ form a solution to problem $(\mathrm{C})$ with $\Gamma$ in $C^{1+\alpha}$, and write

$$
\mathbf{J}(t)=\left(J_{1}(t), J_{2}(t)\right)=\left(J_{1}(X(t)), J_{2}(X(t)) .\right.
$$

For simplicity we shall always assume that

$$
f(0)=f^{\prime}(0)=0
$$

\section{Lemma 7.1}

$$
\mathbf{J}(0)=\frac{\pi\left(1-\nu^{2}\right)}{2 E}\left(36 A_{1}^{2}+4 A_{2}^{2}, 24 A_{1} A_{2}\right),
$$

Proof. Consider first the case of the tip $X(0)$. By Theorem 3.1,

$$
\varphi(x)=A_{1} r^{3 / 2}\left(\cos \frac{3}{2} \theta+3 \cos \frac{1}{2} \theta\right)+A_{2} r^{3 / 2}\left(\sin \frac{3}{2} \theta+\sin \frac{1}{2} \theta\right)+G \equiv r^{3 / 2} \mathbf{A} \cdot \mathbf{B}(\theta)+G,
$$

where

$$
G=O\left(r^{3 / 2+\mu}\right) \quad\left(\text { for any } 0<\mu<\frac{1}{2}\right) .
$$

Since $\Delta^{2} G=0$, by interior elliptic estimates

$$
\left|D^{2} G\right|=O\left(r^{-1 / 2+\mu}\right)
$$

Since $|\Delta \varphi|=O\left(r^{-1 / 2}\right)$, we also have

$$
\left|(\Delta \varphi)^{c}\right|=O\left(r^{-1 / 2}\right),
$$

as can be seen by writing $(\Delta \varphi)^{c}$ as a line integral of $\left((\Delta \varphi)_{y},-(\Delta \varphi)_{x}\right)$.

Similarly

$$
\left|(\Delta G)^{c}\right|=O\left(r^{-1 / 2+\mu}\right) .
$$

From the above estimates we easily conclude that we can take $G \equiv 0$ in the calculation of the $J$-integral. Using complex variables $z=x_{1}+i x_{2}=r e^{i \theta}$, we have

$$
\begin{aligned}
\varphi & =A_{1} r^{3 / 2}\left(\cos \frac{3}{2} \theta+3 \cos \frac{1}{2} \theta\right)+A_{2} r^{3 / 2}\left(\sin \frac{3}{2} \theta+\sin \frac{1}{2} \theta\right), \\
& =C z^{3 / 2}+\bar{C} \bar{z}^{3 / 2}+D z \bar{z}^{1 / 2}+\bar{D} z^{1 / 2} \bar{z}
\end{aligned}
$$

where

$$
C=\frac{1}{2}\left(A_{1}-i A_{2}\right), \quad D=\frac{1}{2}\left(3 A_{1}-i A_{2}\right) .
$$

Then (cf. [8; pp. 275-276])

$$
\begin{aligned}
\Delta \varphi & =6 A_{1} r^{-1 / 2} \cos \frac{1}{2} \theta+2 A_{2} r^{-1 / 2} \sin \frac{1}{2} \theta=2 D \bar{z}^{-1 / 2}+2 \bar{D} z^{-1 / 2}, \\
(\Delta \varphi)^{c} & =-6 A_{1} r^{-1 / 2} \sin \frac{1}{2} \theta+2 A_{2} r^{-1 / 2} \cos \frac{1}{2} \theta=-2 i \bar{D} z^{-1 / 2}+2 i D \bar{z}^{-1 / 2}, \\
\frac{\partial}{\partial \theta}(\Delta \varphi) & =-3 A_{1} r^{-1 / 2} \sin \frac{1}{2} \theta+A_{2} r^{-1 / 2} \cos \frac{1}{2} \theta=-i \bar{D} z^{-1 / 2}+i D \bar{z}^{-1 / 2}, \\
\frac{\partial}{\partial \theta}(\Delta \varphi)^{c} & =-3 A_{1} r^{-1 / 2} \cos \frac{1}{2} \theta-A_{2} r^{-1 / 2} \sin \frac{1}{2} \theta=-D \bar{z}^{-1 / 2}-\bar{D} z^{-1 / 2} .
\end{aligned}
$$


It follows that

$$
\begin{aligned}
\int_{\Lambda}(\Delta \varphi)^{2} n_{1} d l & =\int_{\Lambda}\left[2 D \bar{z}^{-1 / 2}+2 \bar{D} z^{-1 / 2}\right]^{2} \frac{1}{2}\left(e^{i \theta}+e^{-i \theta}\right) d l \\
& =\int_{-\pi}^{\pi}\left[2 D e^{i \theta / 2}+2 \bar{D} e^{-i \theta / 2}\right]^{2} \frac{1}{2}\left(e^{i \theta}+e^{-i \theta}\right) d \theta \\
& =2 \pi\left(2 D^{2}+2 \bar{D}^{2}\right)=18 \pi A_{1}^{2}-2 \pi A_{2}^{2}
\end{aligned}
$$

where $\Lambda$ is the circle $r=\varepsilon,-\pi<\theta<\pi$, traced counterclockwise. Similarly

$$
\begin{aligned}
\int_{\Lambda}(\Delta \varphi)^{2} n_{2} d l & =\int_{\Lambda}\left[2 D \bar{z}^{-1 / 2}+2 \bar{D} z^{-1 / 2}\right]^{2} \frac{1}{2 i}\left(e^{i \theta}+e^{-i \theta}\right) d l \\
& =\int_{-\pi}^{\pi}\left[2 D e^{i \theta / 2}+2 \bar{D} e^{-i \theta / 2}\right]^{2} \frac{1}{2 i}\left(e^{i \theta}+e^{-i \theta}\right) d \theta \\
& =2 \pi i\left(2 D^{2}-2 \bar{D}^{2}\right)=12 \pi A_{1} A_{2} .
\end{aligned}
$$

To evaluate $\vec{s}$, we compute

$$
\begin{aligned}
\vec{s} & =\left(\varphi_{22} n_{1}-\varphi_{12} n_{2},-\varphi_{12} n_{1}+\varphi_{11} n_{2}\right), \\
& =\left(\frac{\partial^{2} \varphi}{\partial x_{2}^{2}} \cos \theta-\frac{\partial^{2} \varphi}{\partial x_{1} \partial x_{2}} \sin \theta,-\frac{\partial^{2} \varphi}{\partial x_{1} \partial x_{2}} \cos \theta+\frac{\partial^{2} \varphi}{\partial x^{2}} \sin \theta\right) \\
& =\left(\frac{1}{r} \frac{\partial}{\partial \theta}\left(\frac{\partial \varphi}{\partial x_{2}}\right),-\frac{1}{r} \frac{\partial}{\partial \theta}\left(\frac{\partial \varphi}{\partial x_{1}}\right)\right)
\end{aligned}
$$

Next we compute $\partial \varphi / \partial x_{2}$ and $\partial \varphi / \partial x_{1}$.

$$
\begin{aligned}
\frac{\partial \varphi}{\partial x_{1}} & =\frac{\partial \varphi}{\partial z}+\frac{\partial \varphi}{\partial \bar{z}} \\
& =\frac{3}{2}\left(C z^{1 / 2}+\bar{C} \bar{z}^{1 / 2}\right)+D \bar{z}^{1 / 2}+\bar{D} z^{1 / 2}+\frac{1}{2}\left(\bar{D} z^{-1 / 2} \bar{z}+D \bar{z}^{-1 / 2} z\right), \\
\frac{\partial \varphi}{\partial x_{2}} & =i\left(\frac{\partial \varphi}{\partial z}-\frac{\partial \varphi}{\partial \bar{z}}\right) \\
& =\frac{3}{2} i\left(C z^{1 / 2}-\bar{C} \bar{z}^{1 / 2}\right)+i\left(D \bar{z}^{1 / 2}-\bar{D} z^{1 / 2}\right)+\frac{1}{2} i\left(\bar{D} z^{-1 / 2} \bar{z}-D \bar{z}^{-1 / 2} z\right) .
\end{aligned}
$$

By integration by parts (both $\partial \varphi / \partial x_{1}$ and $\partial \varphi / \partial x_{2}$ vanishes on $\theta= \pm \pi$ ) we then get

$$
\begin{gathered}
\int_{\Lambda} \vec{s} \cdot \vec{\Phi}_{1} d l=\int_{-\pi}^{\pi}\left(\frac{\partial}{\partial \theta}\left(\frac{\partial \varphi}{\partial x_{2}}\right),-\frac{\partial}{\partial \theta}\left(\frac{\partial \varphi}{\partial x_{1}}\right)\right) \cdot\left(\Delta \varphi,(\Delta \varphi)^{c}\right) d \theta \\
=\int_{-\pi}^{\pi}\left(-\frac{\partial \varphi}{\partial x_{2}}, \frac{\partial \varphi}{\partial x_{1}}\right) \cdot\left(\frac{\partial}{\partial \theta} \Delta \varphi, \frac{\partial}{\partial \theta}(\Delta \varphi)^{c}\right) d \theta \\
=\int_{-\pi}^{\pi}\left(-\frac{\partial \varphi}{\partial x_{2}} \frac{\partial}{\partial \theta} \Delta \varphi+\frac{\partial \varphi}{\partial x_{1}} \frac{\partial}{\partial \theta}(\Delta \varphi)^{c}\right) d \theta \equiv K_{1}+K_{2}
\end{gathered}
$$

Substituting the formulas obtained above into the various expression which appears in the integrand of $K_{1}$, we get

$$
K_{1}=-\int_{-\pi}^{\pi}\left\{\frac{3}{2} i\left(C e^{i \theta / 2}-\bar{C} e^{-i \theta / 2}\right)+i\left(D e^{-i \theta / 2}-\bar{D} e^{i \theta / 2}\right)\right.
$$




$$
\begin{aligned}
& \left.\quad+\frac{1}{2} i\left(\bar{D} e^{-i 3 \theta / 2}-D e^{i 3 \theta / 2}\right)\right\} \cdot\left(-i \bar{D} e^{-i \theta / 2}+i D e^{i \theta / 2}\right) d \theta \\
& =2 \pi\left[-\frac{3}{2} C \bar{D}-\frac{3}{2} \bar{C} D+D^{2}+\bar{D}^{2}\right] .
\end{aligned}
$$

Similarly

$$
\begin{aligned}
K_{2}= & \int_{-\pi}^{\pi}\left\{\frac{3}{2}\left(C e^{i \theta / 2}+\bar{C} e^{-i \theta / 2}\right)+\left(D e^{-i \theta / 2}+\bar{D} e^{i \theta / 2}\right)\right. \\
& \left.\quad+\frac{1}{2}\left(\bar{D} e^{-i 3 \theta / 2}+D e^{i 3 \theta / 2}\right)\right\} \cdot\left(-D e^{i \theta / 2}-\bar{D} e^{-i \theta / 2}\right) d \theta \\
= & 2 \pi\left[-\frac{3}{2} C \bar{D}-\frac{3}{2} \bar{C} D-D^{2}-\bar{D}^{2}\right] .
\end{aligned}
$$

It follows that

$$
K_{1}+K_{2}=6 \pi(C \bar{D}+\bar{C} D)=-3 \pi\left(3 A_{1}^{2}+A_{2}^{2}\right),
$$

and, together with (7.4),

$$
J_{1}=\frac{\pi\left(1-\nu^{2}\right)}{2 E}\left[\left(18 \pi A_{1}^{2}-2 \pi A_{2}^{2}\right)-2\left(K_{1}+K_{2}\right)\right]=\frac{\pi\left(1-\nu^{2}\right)}{2 E}\left(36 A_{1}^{2}+4 A_{2}^{2}\right) .
$$

In a similar way we compute

$$
\begin{gathered}
\int_{\Lambda} \vec{s} \cdot \vec{\Phi}_{2} d l=\int_{-\pi}^{\pi}\left(\frac{\partial}{\partial \theta}\left(\frac{\partial \varphi}{\partial x_{2}}\right),-\frac{\partial}{\partial \theta}\left(\frac{\partial \varphi}{\partial x_{1}}\right)\right) \cdot\left(-(\Delta \varphi)^{c}, \Delta \varphi\right) d \theta \\
\quad=\int_{-\pi}^{\pi}\left(-\frac{\partial \varphi}{\partial x_{2}}, \frac{\partial \varphi}{\partial x_{1}}\right) \cdot\left(-\frac{\partial}{\partial \theta}(\Delta \varphi)^{c}, \frac{\partial}{\partial \theta} \Delta \varphi\right) d \theta \\
=\int_{-\pi}^{\pi}\left(\frac{\partial \varphi}{\partial x_{2}} \frac{\partial}{\partial \theta}(\Delta \varphi)^{c}+\frac{\partial \varphi}{\partial x_{1}} \frac{\partial}{\partial \theta} \Delta \varphi\right) d \theta \equiv \tilde{K}_{1}+\tilde{K}_{2},
\end{gathered}
$$

where

$$
\begin{aligned}
\widetilde{K}_{1}= & \int_{-\pi}^{\pi}\left\{\frac{3}{2} i\left(C e^{i \theta / 2}-\bar{C} e^{-i \theta / 2}\right)+i\left(D e^{-i \theta / 2}-\bar{D} e^{i \theta / 2}\right)\right. \\
& \left.+\frac{1}{2} i\left(\bar{D} e^{-i 3 \theta / 2}-D e^{i 3 \theta / 2}\right)\right\} \cdot\left(-D e^{i \theta / 2}-\bar{D} e^{-i \theta / 2}\right) d \theta \\
= & 2 \pi\left[\frac{3}{2} i \bar{C} D-\frac{3}{2} i C \bar{D}-i D^{2}+i \bar{D}^{2}\right],
\end{aligned}
$$

and

$$
\begin{aligned}
\tilde{K}_{2}= & \int_{-\pi}^{\pi}\left\{\frac{3}{2}\left(C e^{i \theta / 2}+\bar{C} e^{-i \theta / 2}\right)+\left(D e^{-i \theta / 2}+\bar{D} e^{i \theta / 2}\right)\right. \\
& \left.\quad+\frac{1}{2}\left(\bar{D} e^{-i 3 \theta / 2}+D e^{i 3 \theta / 2}\right)\right\} \cdot\left(-i \bar{D} e^{-i \theta / 2}+i D e^{i \theta / 2}\right) d \theta \\
= & 2 \pi\left[-\frac{3}{2} i C \bar{D}+\frac{3}{2} i \bar{C} D-i \bar{D}^{2}+i D^{2}\right] .
\end{aligned}
$$

It follows that

$$
\widetilde{K}_{1}+\widetilde{K}_{2}=6 \pi(i \bar{C} D-i C \bar{D})=-6 \pi A_{1} A_{2},
$$


and, together with (7.5),

$$
J_{2}=\frac{\pi\left(1-\nu^{2}\right)}{2 E}\left[12 A_{1} A_{2}-2\left(\widetilde{K}_{1}+\widetilde{K}_{2}\right)\right]=\frac{\pi\left(1-\nu^{2}\right)}{2 E}\left(24 A_{1} A_{2}\right) .
$$

Remark 7.1. We denote by $\theta_{P}$ the angle from the positive $x$-axis to $\dot{X}(t)$. If we rotate the coordinate system by an angle $\theta_{P}$, then the formula in Lemma 7.1 is valid in the new coordinate system. Therefore we expect the formula for $\mathbf{J}=\left(J_{1}, J_{2}\right)$ in the original coordinate system to be:

$$
\begin{aligned}
& J_{1}=\frac{\pi\left(1-\nu^{2}\right)}{2 E}\left[\left(36 A_{1}^{2}+4 A_{2}^{2}\right) \cos \theta_{P}-24 A_{1} A_{2} \sin \theta_{P}\right], \\
& J_{2}=\frac{\pi\left(1-\nu^{2}\right)}{2 E}\left[\left(36 A_{1}^{2}+4 A_{2}^{2}\right) \sin \theta_{P}+24 A_{1} A_{2} \cos \theta_{P}\right] .
\end{aligned}
$$

We shall now verify (7.10) directly. From the computations in Lemma 7.1, we get,

$$
\mathbf{J}=\int \vec{n} \cdot(\Delta \varphi)^{2}-2 \int\left(\frac{\partial \varphi}{\partial x_{1}}, \frac{\partial \varphi}{\partial x_{2}}\right)\left(\begin{array}{cc}
\frac{\partial}{\partial \theta}(\Delta \varphi)^{c}, & \frac{\partial}{\partial \theta} \Delta \varphi \\
-\frac{\partial}{\partial \theta} \Delta \varphi, & \frac{\partial}{\partial \theta}(\Delta \varphi)^{c}
\end{array}\right) .
$$

Under the new coordinate system $x_{1}^{\prime}=\cos \theta_{P} x_{1}+\sin \theta_{P} x_{2}, x_{2}^{\prime}=-\sin \theta_{P} x_{1}+\cos \theta_{P} x_{2}$, we have

$$
\begin{aligned}
& \vec{n}=(\cos \theta, \sin \theta) \\
&=\left(\cos \left(\theta-\theta_{P}\right) \cos \theta_{P}-\sin \left(\theta-\theta_{P}\right) \sin \theta_{P}, \cos \left(\theta-\theta_{P}\right) \sin \theta_{P}+\sin \left(\theta-\theta_{P}\right) \cos \theta_{P}\right), \\
&=\left(\cos \left(\theta-\theta_{P}\right), \sin \left(\theta-\theta_{P}\right)\right)\left(\begin{array}{cc}
\cos \theta_{P}, & \sin \theta_{P} \\
-\sin \theta_{P}, \cos \theta_{P}
\end{array}\right), \\
&\left(\frac{\partial \varphi}{\partial x_{1}}, \frac{\partial \varphi}{\partial x_{2}}\right)=\left(\frac{\partial \varphi}{\partial x_{1}^{\prime}} \cos \theta_{P}-\frac{\partial \varphi}{\partial x_{2}^{\prime}} \sin \theta_{P}, \frac{\partial \varphi}{\partial x_{1}^{\prime}} \sin \theta_{P}+\frac{\partial \varphi}{\partial x_{2}^{\prime}} \cos \theta_{P}\right) \\
&=\left(\frac{\partial \varphi}{\partial x_{1}^{\prime}}, \frac{\partial \varphi}{\partial x_{2}^{\prime}}\right)\left(\begin{array}{cc}
\cos \theta_{P}, & \sin \theta_{P} \\
-\sin \theta_{P}, & \cos \theta_{P}
\end{array}\right)
\end{aligned}
$$

Clearly

$$
\begin{aligned}
& \left(\begin{array}{cc}
\cos \theta_{P}, & \sin \theta_{P} \\
-\sin \theta_{P}, & \cos \theta_{P}
\end{array}\right)\left(\begin{array}{cc}
\frac{\partial}{\partial \theta}(\Delta \varphi)^{c}, & \frac{\partial}{\partial \theta} \Delta \varphi \\
-\frac{\partial}{\partial \theta} \Delta \varphi, & \frac{\partial}{\partial \theta}(\Delta \varphi)^{c}
\end{array}\right) \\
& =\left(\begin{array}{cc}
\frac{\partial}{\partial \theta}(\Delta \varphi)^{c}, & \frac{\partial}{\partial \theta} \Delta \varphi \\
-\frac{\partial}{\partial \theta} \Delta \varphi, & \frac{\partial}{\partial \theta}(\Delta \varphi)^{c}
\end{array}\right)\left(\begin{array}{cc}
\cos \theta_{P}, & \sin \theta_{P} \\
-\sin \theta_{P}, & \cos \theta_{P}
\end{array}\right),
\end{aligned}
$$

and the 2 matrix with the $\Delta \varphi$ is invariant under the above change of coordinates. Substituting these relations into $\mathbf{J}$, we get (7.10).

Set

$$
\Gamma_{s}=\Gamma_{0} \cup\left\{x_{1}=f\left(x_{2}\right), 0 \leqslant x_{2} \leqslant s\right\}
$$


for any $0 \leqslant s \leqslant s_{0}$ and consider (6.13)-(6.16) with $\Omega \backslash \Gamma(t)$ replaced by $\Omega \backslash \Gamma_{s}$ and with (6.8) replaced by

$$
f^{\prime}(s)=\frac{J_{2}(s)}{J_{1}(s)}
$$

where

$$
\mathbf{J}(s)=\mathbf{J}((s, f(s))
$$

We shall refer to this problem as Problem $\left(\mathrm{C}_{0}\right)$.

Lemma 7.2 Problems (C) and $\left(C_{0}\right)$ are equivalent.

Proof. For a solution $(\varphi, X(t))$ to problem $(\mathrm{C})$, we have

$$
\frac{\dot{X}_{2}(t)}{\dot{X}_{1}(t)}=\frac{J_{2}(X(t))}{J_{1}(X(t))} .
$$

If we write $X_{1}(t)=s, X_{2}(t)=f(s)$, then

$$
f^{\prime}(s)=\frac{\dot{X}_{2}(t)}{\dot{X}_{1}(t)}=\frac{J_{2}((s, f(s))}{J_{1}((s, f(s))}
$$

with $x_{2}=f(s)$ defined by $f(s)=X_{2}\left(X_{1}^{-1}(s)\right)$, which shows that $(\varphi, f(s))$ forms a solution to problem $\left(\mathrm{C}_{0}\right)$.

Conversely, let $\left(\varphi, \Gamma_{s}\right)$ be a solution to problem $\left(\mathrm{C}_{0}\right)$ and define $X(t)=\left(X_{1}(t), X_{2}(t)\right)$ by

$$
\dot{X}(t)=h(|\mathbf{J}(t)|) \mathbf{J}(t)
$$

where $\mathbf{J}(t)=\mathbf{J}(X(t))$. Writing $X_{1}(t)=s$ or $t=X_{1}^{-1}(s)$, we introduce a function $X_{2}=\widehat{f}(s)$ by

$$
\widehat{f}(s)=X_{2}\left(X_{1}^{-1}(s)\right)
$$

Thus

$$
\widehat{f}(s)=\frac{\dot{X}_{2}(t)}{\dot{X}_{1}(t)}=\frac{J_{2}(s, f(s))}{J_{1}(s, f(s))}
$$

which implies that $\widehat{f}^{\prime}$ at $X_{1}(t)$ agrees with $f^{\prime}$ at $s$, i.e., $\widehat{f}$ and $f$ define the same curve with different parameterizations. It follows that the $\mathbf{J}(t)$ in (7.14) is the $J$-integral for the tip $X(t)$ of the curve defined by $f(s), 0 \leqslant s \leqslant X_{1}(t)$; hence $(\varphi, X(t))$ is a solution to problem $(\mathrm{C})$.

We proceed to consider problem $\left(\mathrm{C}_{0}\right)$, and denote by $A_{1}(s), A_{2}(s), \cdots$ the coefficients in the asymptotic expansion of the solution about the tip $X(s)=(x, f(s))$.

From Lemma 7.1, it is clear that

$$
\frac{J_{2}}{J_{1}}=\frac{\tan \theta_{P}+g\left(A_{2} / A_{1}\right)}{1-\tan \theta_{P} g\left(A_{2} / A_{1}\right)}
$$


where

$$
g(u)=\frac{6 u}{9+u^{2}}, \quad g^{\prime}(u)=\frac{54-6 u^{2}}{\left(9+u^{2}\right)^{2}} .
$$

If the curve in problem $\left(\mathrm{C}_{0}\right)$ is given by $(s, f(s))$, then, at $s$,

$$
\tan \theta_{P}=f^{\prime}(s) \text {. }
$$

Substituting this into (7.15) we conclude that (7.12) is equivalent to

$$
g\left(\frac{A_{2}}{A_{1}}\right)=0
$$

or, by (7.16),

$$
\text { either } A_{1}=0 \text { or } A_{2}=0 \text {. }
$$

We shall henceforce assume that

$$
A_{2}(0)=0 \text {. }
$$

Since $|\mathbf{J}(0)|>\gamma_{0}, A_{1}(0)$ is necessarily $\neq 0$ and by continuity (assuming that $|\mathbf{J}(s)|>\gamma_{0}$ ) we get that

$$
A_{2}(s)=0, \quad A_{1}(s) \neq 0 .
$$

In particular:

Theorem $7.3(\varphi, f(s))$ is a solution to problem $\left(C_{0}\right)$ if and only if

$$
A_{2}(s) \equiv 0 \text {. }
$$

Thus the crack problem is equivalent to the following:

Problem $\left(\mathrm{C}_{1}\right)$. Find a pair $(\varphi, f(s))$ such that $\varphi$ satisfies $(6.13)-(6.16)$ with $\Omega \backslash \Gamma$ replaced by $\Omega \backslash \Gamma_{s}, \Gamma_{s}$ as in (7.11), with (7.12) replaced by (7.19).

Condition (7.19) implies that

$$
\varphi=r_{P}^{3 / 2} A_{1}(s) B_{1}\left(\theta-\theta_{P}\right)+O\left(r_{P}^{3 / 2+\lambda}\right) \quad(0<\lambda<1 / 2)
$$

in a neighborhood of the tip $P=(s, f(s))$. Consequently, as we approach $P$ from $\Omega \backslash \Gamma_{s}$ along the tangent $\tau$ to $\Gamma_{s}$ at $P$,

$$
\varphi_{\tau \tau} \sim \frac{K}{r_{P}^{1 / 2}} \quad(K \neq 0), \quad \varphi_{\tau n} \rightarrow 0
$$

where $n$ is the direction normal to $\tau$, or, in terms of the stress $\sigma$,

$$
\sigma_{n n} \sim \frac{K}{r_{P}^{1 / 2}} \quad(K \neq 0), \quad \sigma_{\tau n} \rightarrow 0 .
$$

This local behavior is used by some authors (e.g. [7; p. 433] [4]) to model the propagation of cracks developed by traction (and commonly called mode I, or opening mode ([16; p. 24]).

Since, conversely, (7.20) implies (7.19), we have thus obtained a very interesting physical result:

Theorem 7.4 In the modeling of the crack propagation problem, the conditions (\%.13) and (7.20) are equivalent (assuming the crack is in $C^{1+\alpha}$ ). 


\section{Remarks on problem $\left(C_{0}\right)$}

The results of $\S \S 3,5$ can be used to study the regularity of the coefficients $A_{i}(s)$. As an example, we shall establish in this section the Hölder continuity of $\mathbf{A}(s)=\left(A_{1}(s), A_{2}(s)\right)$. We assume that

$$
\Gamma_{s}: \quad x_{2}=f(s),-1 \leqslant s \leqslant \tau(\tau>0)
$$

is a $C^{1+\alpha}$ curve initiating on $\partial \Omega$ and contained in $\Omega$, with

$$
f(0)=0, \quad f^{\prime}(0)=0,
$$

and set $\Omega_{s}=\Omega \backslash \Gamma_{s}$. Let $\psi(x, s)$ be the solution of

$$
\begin{aligned}
& \psi \in H^{2}\left(\Omega_{s}\right), \\
& \Delta^{2} \psi=0 \quad \text { in } \Omega_{s}, \\
& \psi=\frac{\partial \psi}{\partial n}=0 \quad \text { from both sides of } \Gamma_{s} . \\
& \psi=g, \frac{\partial \psi}{\partial n}=h \quad \text { on } \partial \Omega
\end{aligned}
$$

where $g, h$ are independent of $s$.

By Theorem 3.1, if $X(s)=(s, f(s)), 0 \leqslant s \leqslant \tau$,

$$
\psi(x, s)=|x-X(s)|^{3 / 2} \mathbf{A}(s) \cdot \mathbf{B}\left(\theta-\arctan f^{\prime}(s)\right)+O\left(|x-X(s)|^{2-\eta}\right)
$$

for any $\eta$ such that $\alpha+\eta>1 / 2$.

Set

$$
w(x, s)=\psi(x, s)-\psi(x, 0) \quad \text { for } s>0 .
$$

Lemma 8.1 For any sufficiently large p,

$$
\left(\int_{\Omega_{0}}|w(x, s)|^{p} d x\right)^{1 / p} \leqslant C_{p} s .
$$

Proof. It is clear that, for any $s>0$,

$$
\begin{aligned}
& |\psi(x, s)| \leqslant C|x-(s, f(s))|^{3 / 2}, \\
& \left|\nabla_{x} \psi(x, s)\right| \leqslant C|x-(s, f(s))|^{1 / 2} .
\end{aligned}
$$

It follows that

$$
\begin{aligned}
& |w(x, s)| \leqslant C s^{3 / 2} \quad \text { for }|x-(s, 0)| \leqslant 2 s, \\
& \left|\nabla_{x} w(x, s)\right| \leqslant C s^{1 / 2} \quad \text { for }|x-(s, 0)| \leqslant 2 s .
\end{aligned}
$$

Applying Theorem 2.3 in the domain $\Omega_{0} \cap\{|x-(s, 0)|>s\}$ with $\Gamma_{\delta}$ replaced by $\{|x-(s, 0)|=$ $s\}$, we obtain the assertion (8.8).

We shall use Lemma 8.1 and (8.7) to prove the following: 
Theorem 8.2 Let

$$
\|f\|_{C^{1}} \leqslant F_{1}, \quad\left[f^{\prime}\right]_{C^{\alpha}} \leqslant F_{1, \alpha}
$$

for $0<\alpha<1 / 4$. Then $\mathbf{A}(s)$ is Hölder continuous:

$$
|\mathbf{A}(X(s))-\mathbf{A}(X(\widehat{s}))| \leqslant C(s-\widehat{s})^{\widetilde{\mu}} \quad(0 \leqslant \widehat{s}<s \leqslant \tau)
$$

for any $0<\tilde{\mu}<\alpha / 4$ where $C$ depends only on $\alpha, F_{1}, F_{1, \alpha}$.

Proof. It suffices to take $\widehat{s}=0$. Note that in (8.7),

$$
\left|O\left(|x-X(s)|^{2-\eta}\right)\right| \leqslant C|x-X(s)|^{2-\eta}
$$

where $C$ depends on $F_{1}, F_{1, \alpha}$ but is independent of $s$. It is also clear that $|X(s)| \leqslant C s$. Therefore, for $\sqrt{s} \leqslant|x| \leqslant 2 \sqrt{s}$,

$$
\frac{w(x)}{|x|^{3 / 2}}=\mathbf{A}(X(0)) \cdot \mathbf{B}(\theta)-\mathbf{A}(X(s)) \cdot \mathbf{B}\left(\theta-\arctan f^{\prime}(s)\right)+O\left(s^{\mu}\right), \quad \mu=\frac{1}{2}\left(\frac{1}{2}-\eta\right)
$$

We substitute (8.10) into (8.8) and then integrate over the region $\{\sqrt{s}<|x|<2 \sqrt{s}\}$. By choosing $p$ to be sufficiently large, we conclude that, for any $\tilde{\mu} \in(0, \mu / 2)$,

$$
\left(\int_{-\pi+C s^{\alpha / 2}}^{\pi-C s^{\alpha / 2}}\left|\mathbf{A}(X(s)) \cdot \mathbf{B}\left(\theta-\arctan f^{\prime}(s)\right)-\mathbf{A}(0) \cdot \mathbf{B}(\theta)\right|^{p} d \theta\right)^{1 / p} \leqslant C s^{\widetilde{\mu}} .
$$

Since $\mu$ can be chosen arbitrarily close to (but smaller than) $\alpha / 2, \tilde{\mu}$ can be chosen arbitrarily close to (but smaller than) $\alpha / 4$. Noting that $\left|\mathbf{B}\left(\theta-\arctan f^{\prime}(s)\right)-\mathbf{B}(\theta)\right| \leqslant C\left[f^{\prime}\right]_{\alpha} s^{\alpha},(8.9)$ easily follows.

A simple approach to solving problem $\left(C_{1}\right)$ is to introduce a family of curves

$$
Y=\left\{f(s) \mid f(0)=f^{\prime}(0)=0 ;\left[f^{\prime}\right]_{C^{\alpha}\left[0, s_{0}\right]} \leqslant M_{1, \alpha}\right\}
$$

where $0<\alpha<1 / 4$.

For any $\tilde{f}(s) \in Y$, let $\widetilde{\varphi}(x, s)$ denote the solution of $(6.13)-(6.16)$ with $\Omega \backslash \Gamma(t)$ replaced by $\widetilde{G}_{s}$, where $\widetilde{G}_{s}$ is defined as in (7.11) with $f$ replaced by $\tilde{f}$. Writing

$$
\tilde{\varphi}(x, s)=r_{P}^{3 / 2}\left[\widetilde{A}_{1}(s) B_{1}\left(\theta-\theta_{P}\right)+A_{2}(s) B_{2}\left(\theta-\theta_{P}\right)\right]+O\left(r_{P}^{3 / 2+\lambda}\right)
$$

(where $0<\lambda<\alpha$ ), we introduce the functional

$$
\mathcal{M}(\tilde{f})=\int_{0}^{s_{0}}\left(\widetilde{A}_{2}(s)\right)^{2} d s
$$

and consider the minimization problem:

$$
\min _{\tilde{f} \in Y} \mathcal{M}(\tilde{f})=\mathcal{M}(f), \quad f \in Y .
$$


Since the $\widetilde{A}_{2}(s)$ are uniformly Hölder continuous (by Theorem 8.2 ), a minimizing sequence $\left(\tilde{f}_{n}, \widetilde{A}_{2, n}\right)$ has a uniformly convergent subsequence to a limit $\left(f, A_{2}\right)$. If the minimum in (8.13) is equal to zero, then $A_{2}=0$ and so $f$ is a solution to problem $\left(C_{0}\right)$. Thus we may view (8.13) as a relaxation of the crack propagation problem.

It is not clear how to prove that the minimum in (8.13) is equal to zero. In a future paper, currently under preparation, we shall use the results obtained in the previous sections in order to rewrite the condition $A_{2}(s)=0$ as a relation between the curvature $\kappa(s)$ at $X(s)$ and leading coefficients in the expansions near $X(s)$ of $\psi(x, s)$ and its tangential derivative. This relation should enable us to establish the existence of a solution of problem $\left(C_{0}\right)$.

\section{Appendix: sub-Schauder estimates}

Let $\Omega$ be a 2 -dimensional bounded domain containing the origin, and $\Gamma_{j}(j=1, \cdots, m)$ be $C^{1+\alpha}$ arcs initiating at the origin and contained in $\Omega$. Set $\Gamma=\bigcup_{j=1}^{m} \Gamma_{j}$. For any small $r$ denote by $\omega(r)$ the largest arc on the circle $|x|=r$ which is contained in $\Omega \backslash \Gamma$, and set $\widehat{\omega}=\inf _{0<r<r_{0}} \omega(r)$ for some small $r_{0}>0, \max (\pi, \widehat{\omega})<\widetilde{\omega} \leqslant 2 \pi$. It is easy to verify that there is a unique solution $\delta=\delta(\widetilde{\omega})$ of

$$
\sin ^{2}(\tilde{\omega} \delta)=\delta^{2} \sin ^{2} \tilde{\omega} \quad \text { such that } 0<\tilde{\omega} \delta(\widetilde{\omega}) \leqslant \pi, \quad \delta(\widetilde{\omega}) \geqslant \frac{1}{2} .
$$

Now let $u$ be a solution of

$$
\begin{aligned}
& \Delta^{2} u=f \quad \text { in } \Omega \backslash \Gamma, \quad u \in H^{2}(\Omega \backslash \Gamma), \\
& u=\frac{\partial u}{\partial \nu}=0 \quad \text { from both sides of } \Gamma,
\end{aligned}
$$

where

$$
\int_{\Omega}|f| \leqslant C_{0}
$$

Theorem 9.1 (Kondratév-Oleinik[14],[15]) If $\|u\|_{H^{2}(\Omega \backslash \Gamma)} \leqslant C_{1}$, then the solution $u$ belongs to $C^{1+\delta(\tilde{\omega})}$ in $r_{0}$-neighborhood of the origin, and

$$
\begin{aligned}
& |u(x)| \leqslant C|x|^{1+\delta(\omega)}, \\
& |\nabla u(x)| \leqslant C|x|^{\delta(\omega)},
\end{aligned}
$$

where the constant $C$ depends only on $C_{0}, C_{1}$ and $\Omega$.

Example 1. $\Gamma$ consists of a single $C^{1+\alpha}$ arc with one endpoint at the origin. Theorem 9.1 is then valid with $\delta(\widetilde{\omega})=1 / 2$.

Example 2. $\Gamma$ is a $C^{1+\alpha}$ curve passing through the origin (and $\Gamma=\Gamma_{1} \cup \Gamma_{2}$ ). In this case, $\tilde{\omega}$ can be taken arbitrarily close to $\pi$ (if $r_{0}$ is small enough). Hence $\delta(\tilde{\omega})$ can be taken arbitrarily close to 1 and, in particular, for any $\varepsilon>0$,

$$
\|u\|_{C^{2-\varepsilon}\left\{|x|<r_{0}\right\}} \leqslant C
$$


if $r_{0}$ is small enough; $C$ depends only on the $C^{1+\alpha}$ norm of $\Gamma$ restricted to $\left\{|x|<r_{0}\right\}$ and on bounds on $\int_{\Omega}|f|$ and $\|u\|_{H^{2}(\Omega \backslash \Gamma)}$.

We shall establish a local version of this theorem whereby $\|u\|_{H^{2}(\Omega \backslash \Gamma)}$ is not assumed to be (uniformly) bounded by a constant $C_{1}$ but, instead, $\|u\|_{L^{\infty}(\Omega)}$ is (uniformly) bounded by a constant $C_{1}$. Let $\Omega$ is a 2-dimensional bounded domain, $S$ an open $C^{1+\alpha}$ subarc of $\partial \Omega$ containing the origin in its interior, and

$$
\begin{aligned}
& \Delta^{2} u=f \quad \text { in } \Omega, \quad u \in H^{2}(\Omega), \\
& u=\frac{\partial u}{\partial \nu}=0 \quad \text { on } S, \\
& \int_{\Omega}|f| \leqslant C_{0}, \quad|u| \leqslant C_{1} \quad \text { in } \Omega .
\end{aligned}
$$

Let $\Omega_{0}$ be any subdomain of $\Omega$ such that $\bar{\Omega}_{0} \subset \Omega \cup S$.

Lemma 9.2 If $(9.7)-(9.9)$ hold, then

$$
\|u\|_{H^{2}\left(\Omega_{0}\right)} \leqslant C_{2}
$$

where $C_{2}$ is a constant depending only on $\Omega_{0}, \Omega, S, C_{0}$ and $C_{1}$, and the estimates (9.4) (9.5) hold for any $\delta(\widetilde{\omega})$ arbitrarily close to 1 .

Proof. For simplicity we may assume that the curve $S$ is given by

$$
S: x_{2}=g\left(x_{1}\right), \quad-1<x_{1}<1, \quad g(0)=0
$$

and $\Omega=\left\{x \in B_{1} ; x_{2}>g\left(x_{1}\right)\right\}$ and $\Omega_{0}=B_{1 / 2} \cap \Omega$. For any $0<r \leqslant 1 / 4$, let $\zeta$ be a cutoff function such that

$$
\zeta=1 \quad \text { for }|x|<1-2 r, \quad \zeta=0 \quad \text { for }|x|>1-r, \quad|\nabla \zeta| \leqslant \frac{C}{r}, \quad\left|D^{2} \zeta\right| \leqslant \frac{C}{r^{2}} .
$$

Since $u \in H^{2}(\Omega)$, Theorem 9.1 implies that for any $0<\varepsilon<1, u=O\left(d^{2-\varepsilon}\right), D u=O\left(d^{1-\varepsilon}\right)$ (and then also $D^{2} u=O\left(d^{-\varepsilon}\right)$ and $D^{3} u=O\left(d^{-1-\varepsilon}\right)$ for $|x|<1-r$ where $d=d(x)=$ $\operatorname{dist}(x, S)$. We can therefore integrate by parts

$$
\begin{aligned}
\int_{B_{1}} \zeta^{4} u f & =\int_{B_{1}} \zeta^{4} u \Delta^{2} u=\int_{B_{1}} \Delta\left(\zeta^{4} u\right) \Delta u \\
& =\int_{B_{1}} \zeta^{4}|\Delta u|^{2}+\int_{B_{1}} u \Delta u \Delta \zeta^{4}+2 \int_{B_{1}} \Delta u \nabla u \cdot \nabla \zeta^{4} \\
& =\int_{B_{1}} \zeta^{4}|\Delta u|^{2}+\int_{B_{1}} u \Delta u\left(12 \zeta^{2}|\nabla \zeta|^{2}+4 \zeta^{3} \Delta \zeta\right)+2 \int_{B_{1}} \Delta u \nabla u \cdot\left(4 \zeta^{3} \nabla \zeta\right) \\
& \geqslant \frac{1}{2} \int_{B_{1}} \zeta^{4}|\Delta u|^{2}-\int_{B_{1}} u^{2}\left(12|\nabla \zeta|^{2}+4 \zeta \Delta \zeta\right)^{2}-\int_{B_{1}}|\nabla u|^{2}(8 \zeta|\nabla \zeta|)^{2} .
\end{aligned}
$$


Notice that

$$
\begin{aligned}
\int_{B_{1}} \zeta^{4}|\Delta u|^{2} & =\sum_{i, j} \int_{B_{1}} \zeta^{4} u_{i i} u_{j j} \\
& =\sum_{i, j} \int_{B_{1}} \zeta^{4} u_{i j} u_{i j}+\sum_{i, j} \int_{B_{1}} u_{i i} u_{j} 4 \zeta^{3} \zeta_{j}-\sum_{i, j} \int_{B_{1}} u_{i j} u_{j} 4 \zeta^{3} \zeta_{i} \\
& \geqslant \frac{1}{2} \int_{B_{1}} \zeta^{4}\left|D^{2} u\right|^{2}-C \int_{B_{1}}|\nabla u|^{2} \zeta^{2}|\nabla \zeta|^{2}
\end{aligned}
$$

Combining these two inequalities, and using also the fact that $|u| \leqslant C_{1}$, we obtain

$$
\begin{aligned}
\int_{B_{1}} \zeta^{4}\left|D^{2} u\right|^{2} & \leqslant C C_{1}^{2} \int_{B_{1-r} \backslash B_{1-2 r}}\left(12 \zeta^{2}|\nabla \zeta|^{2}+4 \zeta^{3} \Delta \zeta\right)+C \int_{B_{1-r}}|\nabla u|^{2} \zeta^{2}|\nabla \zeta|^{2} \\
& \leqslant \frac{C C_{1}^{2}}{r^{3}}+\frac{C^{*}}{r^{2}} \int_{B_{1-r}}|\nabla u|^{2}+C_{1} C_{0} .
\end{aligned}
$$

By embedding

$$
\int_{B_{1-r}}|\nabla u|^{2} \leqslant \varepsilon \int_{B_{1-r}}\left|D^{2} u\right|^{2}+\frac{C}{\varepsilon} \int_{B_{1-r}} u^{2},
$$

where the constant $C$ is independent of $\varepsilon$. Taking $\varepsilon=r /\left(32 C^{*}\right)$, we get

$$
\int_{B_{1-2 r}}\left|D^{2} u\right|^{2} \leqslant \int_{B_{1}} \zeta^{4}\left|D^{2} u\right|^{2} \leqslant \frac{C^{* *}\left(C_{1}^{2}+C_{2}^{2}\right)}{r^{3}}+\frac{1}{16} \int_{B_{1-r}}\left|D^{2} u\right|^{2} \quad \forall 0<r \leqslant \frac{1}{4},
$$

which implies that

$$
\int_{B_{1-r}}\left|D^{2} u\right|^{2} \leqslant \frac{16 C^{* *}\left(C_{1}^{2}+C_{2}^{2}\right)}{r^{3}}
$$

Taking $r=1 / 4$ the proof of (9.10) is complete. The conclusions (9.4), (9.5) now follows from Theorem 9.1 (example 2 ) with $\Gamma=S$.

Remark 9.1. The proof of Lemma 9.2 extends to the case where $S$ is replaced by $\Gamma$ as defined at the beginning of this section; i. e., Theorem 9.1 is valid if the assumption $\|u\|_{H^{2}(\Omega \backslash \Gamma)} \leqslant C_{1}$ is replaced by the assumption $|u| \leqslant C_{1}$ in $\Omega$.

The estimate (9.6) is a sub-Schauder estimates for $C^{1+\alpha}$ boundary. The next subSchauder estimates are for $C^{2+\alpha}$ boundary.

Theorem 9.3 Let $\Omega$ be a bounded domain and let $\Omega_{0}$ be a subdomain of $\Omega$ with $\bar{\Omega}_{0} \subset \Omega \cup S$ where $S$ is a $C^{2+\alpha}$ subarc of $\partial \Omega$. Let $u$ be a solution of

$$
\begin{aligned}
& \Delta^{2} u=f \quad \text { in } \Omega, \quad u \in H^{2}(\Omega), \\
& u=g, \quad \frac{\partial u}{\partial \nu}=h \quad \text { on } S .
\end{aligned}
$$

(i) $\left(C^{2+\alpha}\right.$ estimate) If

$$
\|g\|_{C^{2+\alpha}(S)}<\infty, \quad\|h\|_{C^{1+\alpha}(S)}<\infty, \quad \int_{\Omega}|f|^{p} d x<\infty \quad\left(p>\frac{1}{1-\alpha}\right),
$$


then

$$
\|u\|_{C^{2+\alpha}\left(\Omega_{0}\right)} \leqslant C\left(\|g\|_{C^{2+\alpha}(S)}+\|h\|_{C^{1+\alpha}(S)}+\|f\|_{L^{p}(\Omega)}+c_{0}\|u\|_{L^{\infty}(\Omega)}\right)
$$

(ii) $\left(C^{1+\alpha}\right.$ estimate) If

$$
\|g\|_{C^{1+\alpha}(S)}<\infty, \quad\|h\|_{C^{\alpha}(S)}<\infty, \quad \int_{\Omega}|f| d x<\infty
$$

then

$$
\|u\|_{C^{1+\alpha}(\Omega)} \leqslant C\left(\|g\|_{C^{1+\alpha}(S)}+\|h\|_{C^{\alpha}(S)}+\int_{\Omega}|f| d x+c_{0}\|u\|_{L^{\infty}(\Omega)}\right)
$$

if $\Omega_{0}=\Omega$ then the constant $c_{0}$ can be taken to be zero in both cases (i) and (ii).

If $S \in C^{4+\alpha}$ then the result is a consequence of [3; $\left.\S 9\right]$ (which is valid also for n-dimensional domains).

Proof. By subtracting the special solution (2.4), we may assume without loss of generality that $f(x) \equiv 0$. Let $y=\Phi(x)$ be the conformal mapping which flattens the boundary $S$. Under our assumptions, $\Phi \in C^{2+\alpha}$ up to the boundary.

Setting $w(y)=u(x)$, the equation $\Delta^{2} u=0$ becomes

$$
\Delta_{y}\left[k(y) \Delta_{y} w(y)\right]=0
$$

where $k(y)=\left|\nabla_{x} \Phi(x)\right|^{2}$ is in $C^{1+\alpha}$ up to the boundary. Now apply [3; $\left.\S 9\right]$ to immediately conclude both (i) and (ii).

Acknowledgment. The first author is partially supported by National Science Foundation Grant DMS \#9703842. The second and third authors are grateful for a partial support from the Institute for Mathematics and its Application during their visit there. The third author is partially supported by DGICYT Grant PB96-0614.

\section{References}

[1] S. Agmon, The $L^{p}$ approach to the Dirichlet problem, I. Regularity theorems, Ann. Scuola Norm. Sup. Pisa (3) 13 (1959), 405-448.

[2] S. Agmon, Maximum theorems for solutions of higher order elliptic equations, Bull. AMS, 66 (1960), 77-80.

[3] S. Agmon, A. Douglas and L. Nirenberg, Estimates near the boundary for solutions of elliptic partial differential equations satisfying general boundary conditions I, Comm. Pure Appl. Math., 12 (1959), 623-727.

[4] G.J. Barenblatt, Fracture in solids as a free boundary problem, in Free Boundary Problems: Theory and Applications, I.I. Diaz, M.A. Herrero, A. Liñan and J.L. Vazquez eds., Pitman Research Notes in Mathematics, No. 323, Longman, Essex, England (1995), pp. 20-39. 
[5] M. Costabel and M. Dauge, Stable asymptotics for elliptic systems on plane domains with corners, Comm. PDE, 19 (1994), 1677-1726.

[6] M. Dauge, Elliptic Boundary Value Problems on Corner Domains, Lecture Notes in Mathematics, No. 1341, Springer-Verlag, Berlin, 1988.

[7] L.B. Freund, Dynamic Fracture Mechanics, Cambridge University Press, Cambridge, 1990.

[8] A. Friedman, Partial Differential Equations, Holt, Rinehart and Winston, New York, 1969 (Reprinted by Krieger).

[9] A. Friedman and Y. LiU, Propagation of cracks in elastic media, Arch. Rational Mech. Anal., 136 (1996), 235-290.

[10] A.A. Griffith, The phenomenon of rupture and flow in solid, Philos. Trans. Royal. Soc. London, A221 (1920), 163-198.

[11] M. E. GURTIN, On the energy release rate in quasi-static elastic crack propagation, J. Elasticity, 9 (1979), 187-195.

[12] B. HU AND L. WANG, A free boundary problem arising in electrophotography: solutions with connected toner region, SIAM J. Math. Anal., 23 (1992), 1439-1454.

[13] V.A. KondratÉv, Boundary value problems for elliptic equations in domains with conical and angular points, Trans. Moscow Math. Soc., 16 (1967), 209-292.

[14] V.A. Kondratév AND O.A. OlEINIK, Unimprovable estimates in Hölder spaces for generalized solutions of the biharmonic equation, the Navier-Stokes system of equations, and the Von Karman system in non-smooth two-dimensional domains, Vestnik Moskovskogo Universiteta, Matematika, 38 (1983), 22-39.

[15] V.A. KondratÉv AND O.A. OlEINIK, Boundary value problems for partial differential equations in non-smooth domains, Uspekh, Mat. Nauk, 38 (1983), 3-76.

[16] B. Lawn, Fracture of Brittle Solids, second edition, Cambridge University Press, Cambridge, 1993.

[17] MaZ'Ya AND B.A. Plameneveskit, Estimates in $L_{p}$ and in Hölder spaces and the Miranda-Agmon maximum principle for solutions of elliptic boundary value problems with singular points at the boundary, Math. Nachrichten, 81 (1978), 25-82; English transl., Amer. Math. Soc. Transl. (2) 123 (1984), 1-56.

[18] C. Miranda, Teorema del massimo modulo e teorema di esistenza e di unicita per it problema di Dirichlet in due variabili, Annali di Matematica (4), 46 (1958), 265-311. 
[19] J.L. RICE, Mathematical analysis in mechanics of fracture, in Fracture, Vol. 2, ed/ H. Liebowitz, Academic Press, New York, (1968), 191-311.

[20] L.I. Slepyan, Principle of maximum energy dissipation rate in crack dynamics, J. Mech. Phys. Solids, 41 (1993), 1019-1033.

[21] H. STUMP AND K.C. LE, Variational formulation of crack problem for an elastoplastic body at finite strain, Z. Angew. Math. Mech., 72 (1992), 387-396.

[22] D. Vasilopoulos, On the determination of higher order terms of singular elastic stress fields near corners, Numer. Math., 53 (1988), 51-95. 